Old Dominion University

ODU Digital Commons

Physics Faculty Publications

Physics

2021

\title{
Two- and Three-Nucleon Contact Interactions and Ground-State Energies of Light- and Medium-Mass Nuclei
}

\author{
R. Schiavilla \\ Old Dominion University, rschiavi@odu.edu \\ L. Girlanda
}

A. Gnech

A. Kievsky

A. Lovato

See next page for additional authors

Follow this and additional works at: https://digitalcommons.odu.edu/physics_fac_pubs

Part of the Atomic, Molecular and Optical Physics Commons, and the Nuclear Commons

\section{Original Publication Citation}

Schiavilla, R., Girlanda, L., Gnech, A., Kievsky, A., Lovato, A., Marcucci, L. E., Piarulli, M., \& Viviani, M. (2021). Two- and three-nucleon contact interactions and ground-state energies of light- and medium-mass nuclei. Physical Review C, 103(5), 1-18, Article 054003. https://doi.org/10.1103/PhysRevC.103.054003

This Article is brought to you for free and open access by the Physics at ODU Digital Commons. It has been accepted for inclusion in Physics Faculty Publications by an authorized administrator of ODU Digital Commons. For more information, please contact digitalcommons@odu.edu. 


\section{Authors}

R. Schiavilla, L. Girlanda, A. Gnech, A. Kievsky, A. Lovato, L. E. Marcucci, M. Piarulli, and M. Viviani 


\title{
Two- and three-nucleon contact interactions and ground-state energies of light- and medium-mass nuclei
}

\author{
R. Schiavilla $\odot,{ }^{1,2}$ L. Girlanda, ${ }^{3,4}$ A. Gnech $\odot,{ }^{2,5}$ A. Kievsky $\odot,{ }^{6}$ A. Lovato $\odot,{ }^{7,8}$ L. E. Marcucci, ${ }^{5,6}$ M. Piarulli, ${ }^{9}$ and M. Viviani ${ }^{6}$ \\ ${ }^{1}$ Department of Physics, Old Dominion University, Norfolk, Virginia 23529, USA \\ ${ }^{2}$ Theory Center, Jefferson Laboratory, Newport News, Virginia 23606, USA \\ ${ }^{3}$ Department of Mathematics and Physics, University of Salento, 73100 Lecce, Italy \\ ${ }^{4}$ INFN-Lecce, 73100 Lecce, Italy \\ ${ }^{5}$ Department of Physics, University of Pisa, 56127 Pisa, Italy \\ ${ }^{6}$ INFN-Pisa, 56127 Pisa, Italy \\ ${ }^{7}$ Physics Division, Argonne National Laboratory, Argonne, Illinois 60439, USA \\ ${ }^{8}$ INFN-TIFPA Trento Institute of Fundamental Physics and Applications, 38123 Trento, Italy \\ ${ }^{9}$ Department of Physics, Washington University in St. Louis, St. Louis, Missouri 63130, USA
}

(Received 3 February 2021; accepted 6 May 2021; published 24 May 2021)

\begin{abstract}
Classes of two-nucleon $(2 N)$ contact interactions are developed in configuration space at leading order (LO), next-to-leading order (NLO), and next-to-next-to-next-to-leading order (N3LO) by fitting the experimental singlet $n p$ scattering length and deuteron binding energy at LO, and $n p$ and $p p$ scattering data in the laboratoryenergy ranges $0-15 \mathrm{MeV}$ at $\mathrm{NLO}$ and $0-25 \mathrm{MeV}$ at N3LO. These interactions are regularized by including two Gaussian cutoffs, one for $T=0$ and the other for $T=1$ channels. The cutoffs are taken to vary in the ranges $R_{0}=(1.5-2.3) \mathrm{fm}$ and $R_{1}=(1.5-3.0) \mathrm{fm}$. The 780 (1100) data points up to 15 (25) MeV energy, primarily differential cross sections, are fitted by the NLO (N3LO) models with a $\chi^{2} /$ datum about 1.7 or less (well below 1.5), when harder cutoff values are adopted. As a first application, we report results for the binding energies of nuclei with mass numbers $A=3-6$ and 16 obtained with selected LO and NLO $2 N$ models both by themselves as well as in combination with a LO three-nucleon $(3 N)$ contact interaction. The latter is characterized by a single low-energy constant that is fixed to reproduce the experimental ${ }^{3} \mathrm{H}$ binding energy. The inclusion of the $3 \mathrm{~N}$ interaction largely removes the sensitivity to cutoff variations in the few-nucleon systems and leads to predictions for the ${ }^{3} \mathrm{He}$ and ${ }^{4} \mathrm{He}$ binding energies that cluster around 7.8 and $30 \mathrm{MeV}$, respectively. However, in ${ }^{16} \mathrm{O}$ this cutoff sensitivity remains rather strong. Finally, predictions at LO only are also reported for medium-mass nuclei with $A=40,48$, and 90 .
\end{abstract}

DOI: 10.1103/PhysRevC.103.054003

\section{INTRODUCTION}

Understanding the interactions among the constituents of atomic nuclei lies at the heart of nuclear physics and is still a subject of intense research. Since the advent of the effective field theory (EFT) paradigm $[1,2]$ in the early nineties, two-nucleon $(2 N)$ chiral interactions have been developed up to fourth (N3LO) and, more recently, fifth (N4LO) order in the low-energy expansion [3-13]. These interactions provide an accurate description of $2 \mathrm{~N}$ scattering data up to the pion production threshold, comparable to that obtained by phenomenological models [14-16].

Implicit in the definition of an EFT is a cutoff scale $\Lambda$ which marks the separation between the domain of applicability and high-energy scales that characterize processes unresolved by the EFT and whose effects are subsumed in the values of low-energy constants (LECs). An interesting version of EFT is one in which the cutoff is taken to be smaller than the pion mass, that is, the pion mass represents the heavy scale. In such a regime, pions are integrated out and the theory only consists of contact terms between two or more nucleons-pionless EFT $[17,18]$ ( $\not$ EFT). A natural question to ask is how well (or how poorly) will low-energy nuclear structure, including binding energies, charge radii, and magnetic moments, be accounted for by this simpler EFT. As a first step in our attempt to answer this question, we construct in this paper coordinate-space $2 \mathrm{~N}$ contact interactions from fits to scattering observables in a limited range of energies. These $2 N$ interactions are complemented by a LO threenucleon $(3 N)$ contact interaction, constrained to reproduce the ${ }^{3} \mathrm{H}$ binding energy. A first set of calculations of the groundstate energies of the hydrogen and helium isotopes, ${ }^{6} \mathrm{Li}$, and ${ }^{16} \mathrm{O}\left({ }^{40} \mathrm{Ca},{ }^{48} \mathrm{Ca}\right.$, and $\left.{ }^{90} \mathrm{Zr}\right)$ is presented with selected models at LO and NLO (LO only). Results for the same observable with the N3LO models are limited to the ${ }^{3} \mathrm{H},{ }^{3} \mathrm{He} /{ }^{4} \mathrm{He} /{ }^{6} \mathrm{He}$, and ${ }^{6} \mathrm{Li}$ nuclei.

In the $2 N$ system, $\pi$ EFT reduces to the effective range expansion [19]. Due to the unnaturally large values of the $2 \mathrm{~N}$ scattering lengths, it is convenient, in order to extend the domain of applicability of the theory, to consider the inverse 
scattering length as a soft scale [20-22]. As a consequence, this EFT corresponds to an expansion around the unitary limit of infinite scattering length [23,24]. By introducing a single expansion parameter, the ratio of the interaction range to the scattering length [25-28], such a theory accounts for universal phenomena, such as the Efimov effect [29-32], in systems of three and more nucleons.

Depending on the renormalization conditions, two lowenergy counting schemes can consistently be implemented [33]: the Weinberg counting, in which the magnitude of the LECs entering the interaction follows naive dimensional analysis [34,35], and the Kaplan, Savage, and Wise (KSW) counting [20,21], in which their importance is enhanced. In the present paper, we adhere to Weinberg counting (for related work based on KSW counting, see Refs. [36,37]). This implies a certain amount of fine-tuning of the two leading LECs, which have a direct connection to the unnaturally large values of the singlet and triplet scattering lengths. As a matter of fact, we are led to introduce two different cutoffs in the $T=0$ and $T=1$ isospin channels, in order to reduce, in the fitting procedure, the correlations induced by such fine tuning. Following common practice in the construction of $2 N$ interaction models from EFT, we choose to perform an implicit renormalization of the LECs, through the fitting of low-energy experimental data. Had we chosen to fix each one of the two leading LECs to a single observable, like the corresponding scattering length, we would have obtained a dependence (running) on the associated cutoff (or renormalization point), one from each renormalization condition. Since cutoff independence in the description of other observables is to be expected only up to neglected orders, the implicit renormalization procedure is likely to drive the LECs away from the renormalization group running, except around some special value of the cutoff, which needs not be the same for the two leading LECs. It is expected that, when higher orders are included, the optimal cutoff regions will grow until a plateau is realized, and eventually will overlap. We should also mention that at least two independent cutoffs were found to be necessary in order to derive the rules of Weinberg counting from the Wilsonian renormalization group [38].

The present paper is organized as follows. In Sec. II, the $2 N$ contact interaction is introduced up to $\mathrm{N}_{3} \mathrm{LO}^{1}$ and is regularized to obtain its coordinate space representation. In Sec. III, the associated LECs are determined through an order-by-order fit to $2 \mathrm{~N}$ scattering observables below 15 and $25 \mathrm{MeV}$ laboratory energies at, respectively, NLO and N3LO, and to the deuteron binding energy. In Sec. IV, results for the binding energies of ${ }^{3} \mathrm{H},{ }^{3} \mathrm{He},{ }^{4} \mathrm{He},{ }^{6} \mathrm{Li},{ }^{6} \mathrm{He}$, and ${ }^{16} \mathrm{O}$ are reported for selected models at LO and NLO and for the binding energies of ${ }^{40} \mathrm{Ca},{ }^{48} \mathrm{Ca}$, and ${ }^{90} \mathrm{Zr}$ with selected LO models only. The calculations are carried out with hyperspherical-harmonics (HH) methods in systems with mass number $3 \leqslant A \leqslant 6$ and

\footnotetext{
${ }^{1}$ We denote the various orders in the expansion of the interaction following the usual convention in pionfull EFT, where NLO is $O\left(Q^{2}\right)$ suppressed relative to LO and N3LO is $O\left(Q^{2}\right)$ suppressed relative to NLO. Here $Q$ denotes a low-momentum scale.
}

with auxiliary-field diffusion Monte Carlo (AFDMC) methods in $A \geqslant 16$. Finally, a brief summary and some concluding remarks are given in Sec. V.

\section{CONTACT INTERACTIONS AT LO, NLO, AND N3LO: A SUMMARY}

The structure of two-nucleon $(2 N)$ contact interactions at LO, NLO, and N3LO is well known [6]; we provide a brief summary here for completeness. These interactions consist of charge-independent (CI) terms at LO, NLO, and N3LO and charge-dependent (CD) ones at NLO and N3LO. However, in a departure from common practice (at least in the context of pionless EFT), we require the LO interactions to only act in even partial waves. We explain the rationale for such a choice in Sec. IV below.

\section{A. Contact interactions in momentum space}

The interactions in momentum space are listed below order by order in the power counting ( $Q$ denotes generically a low-momentum scale). The momenta $\mathbf{k}$ and $\mathbf{K}$ are defined as $\mathbf{k}=\mathbf{p}^{\prime}-\mathbf{p}$ and $\mathbf{K}=\left(\mathbf{p}^{\prime}+\mathbf{p}\right) / 2$, where $\mathbf{p}$ and $\mathbf{p}^{\prime}$ are the initial and final relative momenta of the two nucleons, and $\sigma_{i}$ and $\boldsymbol{\tau}_{i}$ denote respectively the Pauli spin and isospin operators:

(1) $\mathrm{CI}$ terms of $\mathrm{LO}\left(Q^{0}\right)$ :

$$
v_{\mathrm{LO}}^{\mathrm{CI}}=C_{01} P_{0}^{\sigma} P_{1}^{\tau}+C_{10} P_{1}^{\sigma} P_{0}^{\tau},
$$

where $P_{0}^{\sigma}\left(P_{0}^{\tau}\right)$ and $P_{1}^{\sigma}\left(P_{1}^{\tau}\right)$ are spin (isospin) projection operators on pairs with $S(T)$ equal to 0 and 1 ,

$$
P_{0}^{\sigma}=\frac{1-\sigma_{1} \cdot \sigma_{2}}{4}, \quad P_{1}^{\sigma}=\frac{3+\sigma_{1} \cdot \sigma_{2}}{4},
$$

and similarly for $P_{0}^{\tau}$ and $P_{1}^{\tau}$;

(2) CI term of $\operatorname{NLO}\left(Q^{2}\right)$ :

$$
\begin{aligned}
v_{\mathrm{NLO}}^{\mathrm{CI}}(\mathbf{k}, \mathbf{K})= & C_{1} k^{2}+C_{2} k^{2} \boldsymbol{\tau}_{1} \cdot \boldsymbol{\tau}_{2}+C_{3} k^{2} \boldsymbol{\sigma}_{1} \cdot \boldsymbol{\sigma}_{2} \\
& +C_{4} k^{2} \boldsymbol{\sigma}_{1} \cdot \boldsymbol{\sigma}_{2} \boldsymbol{\tau}_{1} \cdot \boldsymbol{\tau}_{2}+C_{5} S_{12}(\mathbf{k}) \\
& +C_{6} S_{12}(\mathbf{k}) \boldsymbol{\tau}_{1} \cdot \boldsymbol{\tau}_{2}+i C_{7} \mathbf{S} \cdot(\mathbf{K} \times \mathbf{k}),
\end{aligned}
$$

where $S_{12}(\mathbf{k})=3 \sigma_{1} \cdot \mathbf{k} \sigma_{2} \cdot \mathbf{k}-k^{2} \sigma_{1} \cdot \sigma_{2} ;$

(3) CI terms of $\mathrm{N} 3 \mathrm{LO}\left(Q^{4}\right)$ :

$$
\begin{aligned}
v_{\mathrm{N} 3 \mathrm{LO}}^{\mathrm{CI}}(\mathbf{k}, \mathbf{K})= & D_{1} k^{4}+D_{2} k^{4} \boldsymbol{\tau}_{1} \cdot \boldsymbol{\tau}_{2}+D_{3} k^{4} \boldsymbol{\sigma}_{1} \cdot \boldsymbol{\sigma}_{2} \\
& +D_{4} k^{4} \boldsymbol{\sigma}_{1} \cdot \boldsymbol{\sigma}_{2} \boldsymbol{\tau}_{1} \cdot \boldsymbol{\tau}_{2}+D_{5} k^{2} S_{12}(\mathbf{k}) \\
& +D_{6} k^{2} S_{12}(\mathbf{k}) \boldsymbol{\tau}_{1} \cdot \boldsymbol{\tau}_{2}+i D_{7} k^{2} \mathbf{S} \\
& \cdot(\mathbf{K} \times \mathbf{k})+i D_{8} k^{2} \mathbf{S} \cdot(\mathbf{K} \times \mathbf{k}) \boldsymbol{\tau}_{1} \cdot \boldsymbol{\tau}_{2} \\
& +D_{9}[\mathbf{S} \cdot(\mathbf{K} \times \mathbf{k})]^{2}+D_{10}(\mathbf{K} \times \mathbf{k})^{2} \\
& +D_{11}(\mathbf{K} \times \mathbf{k})^{2} \boldsymbol{\sigma}_{1} \cdot \boldsymbol{\sigma}_{2},
\end{aligned}
$$

where $\mathbf{S}=\left(\sigma_{1}+\sigma_{2}\right) / 2$;

(4) $\mathrm{CD}$ terms of $\mathrm{NLO}\left(Q^{2}\right)$ :

$$
v_{\mathrm{NLO}}^{\mathrm{CD}}=C_{0}^{\mathrm{IT}} T_{12} \text {, }
$$

where $T_{12}=3 \tau_{1 z} \tau_{2 z}-\boldsymbol{\tau}_{1} \cdot \boldsymbol{\tau}_{2}$ is the isotensor operator; 
TABLE I. Cutoff values corresponding to models a-d and o.

\begin{tabular}{lccccc}
\hline \hline Model & $\mathrm{a}$ & $\mathrm{b}$ & $\mathrm{c}$ & $\mathrm{d}$ & $\mathrm{o}$ \\
\hline$R_{0}(\mathrm{fm})$ & 1.7 & 1.9 & 2.1 & 2.3 & 1.54592984 \\
$R_{1}(\mathrm{fm})$ & 1.5 & 2.0 & 2.5 & 3.0 & 1.83039397 \\
\hline \hline
\end{tabular}

(5) $\mathrm{CD}$ terms of $\mathrm{N} 3 \mathrm{LO}\left(Q^{4}\right)$ :

$$
\begin{aligned}
v_{\mathrm{N} 3 \mathrm{LO}}^{\mathrm{CD}}(\mathbf{k}, \mathbf{K})= & {\left[C_{1}^{\mathrm{IT}} k^{2}+C_{2}^{\mathrm{IT}} k^{2} \sigma_{1} \cdot \sigma_{2}+C_{3}^{\mathrm{IT}} S_{12}(\mathbf{k})\right.} \\
& \left.+i C_{4}^{\mathrm{IT}} \mathbf{S} \cdot(\mathbf{K} \times \mathbf{k})\right] T_{12} .
\end{aligned}
$$

We note that at N3LO there are four additional CI terms. Following Ref. [39], we have dropped them, since they lead to operator structures in configuration space which depend quadratically on the relative momentum operator and are difficult to implement in quantum Monte Carlo calculations. Their inclusion was shown to lead to no improvement in the fit to the $2 N$ database [39]. As a matter of fact, three combinations of such terms vanish off the energy shell [13] and their effect can be absorbed into a redefinition of the $3 N$ interaction [40]. We have also ignored five additional charge-symmetry-breaking (CSB) terms (one at $\mathrm{NLO}$ and four at $\mathrm{N} 3 \mathrm{LO}$ ) in the $\mathrm{CD}$ sector. There is only a single observable sensitive to these terms, the difference between the $p p$ and $n n$ scattering lengths. Since the interactions at NLO and N3LO without CSB already give $n n$ scattering lengths reasonably close to the empirical value (as shown below), we have made no attempt in constraining the associated LECs and have therefore set them to zero.

\section{B. Regularization and contact interactions in configuration space}

The contact interactions are regularized by multiplying each term by a Gaussian cutoff depending only on the momentum transfer $k$ but which differentiates between the pair isospin $T=0$ and $T=1$ channels, that is,

$$
\begin{aligned}
\widetilde{C}(k) & =e^{-R_{0}^{2} k^{2} / 4} P_{0}^{\tau}+e^{-R_{1}^{2} k^{2} / 4} P_{1}^{\tau} \\
& \longrightarrow C(r)=C_{0}(r) P_{0}^{\tau}+C_{1}(r) P_{1}^{\tau}, \\
C_{\alpha}(r) & =\frac{1}{\pi^{3 / 2} R_{\alpha}^{3}} e^{-\left(r / R_{\alpha}\right)^{2}} .
\end{aligned}
$$

We have investigated five different combinations of $R_{0} / R_{1}$ as listed in Table I and have designated them as models a, b, $\mathrm{c}, \mathrm{d}$, and $\mathrm{o}$. For this latter model (o stands for optimized), the cutoffs have been determined by constraining them along with the LECs $C_{01}$ and $C_{10}$ in a LO fit designed to reproduce the $n p$ effective range expansions (including scattering lengths and effective radii) in $S / T=0 / 1$ and $1 / 0$. We also note that the relationship between the cutoff $\Lambda_{\alpha}$ in momentum space and the cutoff $R_{\alpha}$ in coordinate space is $\Lambda_{\alpha}=2 / R_{\alpha}$ (with $\alpha=0$ or 1), and so $\Lambda_{0}$ and $\Lambda_{1}$ vary in the ranges (172-263) MeV and (132-263) MeV as $R_{0}$ and $R_{1}$ decrease from 2.3 to $1.5 \mathrm{fm}$ and from 3.0 to $1.5 \mathrm{fm}$, respectively.

The coordinate-space representation of the interaction is written as

$$
v=v^{\mathrm{EM}}+v^{\mathrm{CI}}+v^{\mathrm{CD}},
$$

where $v^{\mathrm{EM}}$ is the electromagnetic component, and

$$
\begin{aligned}
& v^{\mathrm{CI}}=v_{\mathrm{LO}}^{\mathrm{CI}}+v_{\mathrm{NLO}}^{\mathrm{CI}}+v_{\mathrm{N} 3 \mathrm{LO}}^{\mathrm{CI}}=\sum_{l=1}^{11} v^{l}(r) O_{12}^{l}, \\
& v^{\mathrm{CD}}=v_{\mathrm{NLO}}^{\mathrm{CD}}+v_{\mathrm{N} 3 \mathrm{LO}}^{\mathrm{CD}}=\sum_{l=12}^{15} v^{l}(r) O_{12}^{l} .
\end{aligned}
$$

The various operator structures of the $\mathrm{CI}$ and $\mathrm{CD}$ components read

$$
\begin{aligned}
& O_{12}^{l=1, \ldots, 11}=\mathbf{1}, \boldsymbol{\tau}_{1} \cdot \boldsymbol{\tau}_{2}, \boldsymbol{\sigma}_{1} \cdot \boldsymbol{\sigma}_{2}, \boldsymbol{\sigma}_{1} \cdot \boldsymbol{\sigma}_{2} \boldsymbol{\tau}_{1} \cdot \boldsymbol{\tau}_{2}, S_{12}, S_{12} \boldsymbol{\tau}_{1} \\
& \cdot \boldsymbol{\tau}_{2}, \mathbf{L} \cdot \mathbf{S}, \mathbf{L} \cdot \mathbf{S} \boldsymbol{\tau}_{1} \cdot \boldsymbol{\tau}_{2},(\mathbf{L} \cdot \mathbf{S})^{2}, \mathbf{L}^{2}, \mathbf{L}^{2} \boldsymbol{\sigma}_{1} \cdot \boldsymbol{\sigma}_{2},
\end{aligned}
$$

and

$$
O_{12}^{l=12, \ldots, 15}=T_{12}, \sigma_{1} \cdot \sigma_{2} T_{12}, S_{12} T_{12}, \mathbf{L} \cdot \mathbf{S} T_{12},
$$

where $S_{12}$ and $\mathbf{L}$ denote the tensor and orbital angular momentum operators, respectively. Hereafter, we will refer to these operators as

$$
l=1, \ldots, 15 \longrightarrow l=c, \tau, \sigma, \sigma \tau, t, t \tau, b, b \tau, b b, q, q \sigma,
$$$$
T, \sigma T, t T, b T \text {. }
$$

We note that $v^{\mathrm{EM}}$ includes the complete electromagnetic interaction up to terms quadratic in the fine structure constant (first- and second-order Coulomb, Darwin-Foldy, vacuum polarization, and magnetic moment terms); see Ref. [15] for explicit expressions. The radial functions $v^{l}(r)$ multiplying the operators $O_{12}^{l}$ are given in Ref. [11] and reported in Appendix A for completeness. Because of the regularization scheme we have adopted, these functions have an implicit dependence on the isospin $T$ of the pair.

\section{FITS TO THE DATABASE}

The (configuration-space) LO, NLO, and N3LO interactions are defined as

$$
\begin{aligned}
v_{\mathrm{LO}} & =v^{\mathrm{EM}}+v_{\mathrm{LO}}^{\mathrm{CI}}, \\
v_{\mathrm{NLO}} & =v^{\mathrm{EM}}+v_{\mathrm{LO}}^{\mathrm{CI}}+v_{\mathrm{NLO}}^{\mathrm{CI}}+v_{\mathrm{NLO}}^{\mathrm{CD}}, \\
v_{\mathrm{N} 3 \mathrm{LO}} & =v^{\mathrm{EM}}+v_{\mathrm{LO}}^{\mathrm{CI}}+v_{\mathrm{NLO}}^{\mathrm{CI}}+v_{\mathrm{N} 3 \mathrm{LO}}^{\mathrm{CI}}+v_{\mathrm{NLO}}^{\mathrm{CD}}+v_{\mathrm{N} 3 \mathrm{LO}}^{\mathrm{CD}},
\end{aligned}
$$

where, as already noted, the full EM interaction is retained at each order (and in all partial waves). At each order, the values of cutoffs that are considered are those listed in Table I. The LO interaction involves two LECs, the NLO interaction has seven additional LECs in the CI sector and one LEC in the CD sector, and the N3LO interaction uses further eleven and four LECs in the CI and CD sectors, respectively. As per the operator structure, $v_{\mathrm{LO}}^{\mathrm{CI}}$ involves the four operators $c, \tau$, $\sigma$, and $\sigma \tau ; v_{\mathrm{NLO}}^{\mathrm{CI}}$ and $v_{\mathrm{NLO}}^{\mathrm{CD}}$ involve, respectively, the seven operators $c, \tau, \sigma, \sigma \tau, t, t \tau$, and $b$, and the single operator $T ; v_{\mathrm{N} 3 \mathrm{LO}}^{\mathrm{CI}}$ and $v_{\mathrm{N} 3 \mathrm{LO}}^{\mathrm{CD}}$ involve, respectively, the eleven operators $c, \tau, \sigma, \sigma \tau, t, t \tau, b, b \tau, b b, q$, and $q \sigma$, and the four operators $T, \sigma T, t T b T$. However, because of the isospin dependence of the radial functions $v^{l}(r)$, the interactions $v_{\mathrm{NLO}}^{\mathrm{CI}}$ 
TABLE II. The LO LECs determined by reproducing the $n p$ singlet scattering length and deuteron binding energy as obtained for models $\mathrm{a}-\mathrm{d}$; for model $\mathrm{o}$, the cutoff radii along with the LO LECs have been constrained by fitting the $n p$ scattering lengths and effective radii in the singlet and triplet channels, and the deuteron binding energy.

\begin{tabular}{lcccrr}
\hline \hline Model & $\mathrm{a}$ & $\mathrm{b}$ & $\mathrm{c}$ & $\mathrm{d}$ & $\mathrm{o}$ \\
\hline$C_{01}\left(\mathrm{fm}^{2}\right)$ & $-0.438524414 \times 10^{1}$ & $-0.572220536 \times 10^{1}$ & $-0.700250932 \times 10^{1}$ & $-0.822926713 \times 10^{1}$ & $-0.527518671 \times 10^{1}$ \\
$C_{10}\left(\mathrm{fm}^{2}\right)$ & $-0.800783936 \times 10^{1}$ & $-0.934392090 \times 10^{1}$ & $-0.107734100 \times 10^{2}$ & $-0.122993164 \times 10^{2}$ & $-0.704040080 \times 10^{1}$ \\
\hline \hline
\end{tabular}

and $v_{\mathrm{N} 3 \mathrm{LO}}^{\mathrm{CI}}$ effectively also include, respectively, the $b \tau$ operator and the $\boldsymbol{\tau}_{1} \cdot \boldsymbol{\tau}_{2}$-dependent $b b, q$, and $q \sigma$ operators. Lastly, the values adopted for the proton and neutron masses are, respectively, 938.27192 and $939.56524 \mathrm{MeV}$, and $\hbar c$ is taken as 197.32697 MeV fm.

The two LECs in the LO interactions are determined by reproducing the singlet $n p$ scattering length $\left({ }^{1} a_{n p}\right)$ and the deuteron binding energy $\left(B_{d}\right)$ in models a-d. In model o, the cutoff radii along with the LO LECs have been constrained by fitting the $n p$ scattering lengths and effective radii in the singlet and triplet channels, and the deuteron binding energy. Their values are listed in Table II. The NLO and N3LO interactions are fitted to $n p$ and $p p$ scattering data (including normalizations), as assembled in the Granada database [41-43], over the laboratory energy range $0-15 \mathrm{MeV}$ at NLO and $0-25 \mathrm{MeV}$ at N3LO, and, simultaneously, to $B_{d}$. The corresponding LECs are reported in Table XIV at NLO and Table XV at N3LO, in Appendix B. The optimization of the objective function $\chi^{2}$ with respect to the LECs is carried out with the Practical Optimization Using No Derivatives (for Squares), POUNDERS [44]. We list the numbers of $n p, p p$, and $n p+p p$ data (including normalizations) and corresponding $\chi^{2} /$ datum for all models in Table III. The NLO and N3LO fits are optimized by minimizing the $\chi^{2}$ corresponding to the total number of $n p+p p$ data. The numbers of data points change slightly for each of the various models because of fluctuations in the number of normalizations; see Ref. [11] for more details on the fitting procedure. Finally, in Table III we also report the $\chi^{2} /$ datum to the $n p$ data in the laboratory energy range $0-1 \mathrm{MeV}$ for the LO models. We stress that these $\chi^{2}$ values do not result from fits but rather correspond to the sets of LECs as determined in Table II. We do not report the $\chi^{2} /$ datum values relative to the $p p$ data, since they are in the thousands to tens of thousands (the number of $p p$ data points in $0-1 \mathrm{MeV}$ is about 160) and therefore meaningless.

The $\chi^{2}$ improves slightly or remains essentially unchanged in going from NLO to N3LO, although the number of data points included in the fits increases from about 780 at $15 \mathrm{MeV}$ to about 1100 at $25 \mathrm{MeV}$; the $\chi^{2}$ improvement is drastic, by about a factor of 2 for model $\mathrm{d}$, corresponding to $R_{0} / R_{1}=$ 2.3/3.0 fm. But for this model, all $\chi^{2}$ at N3LO are well below 2 . Even in the limited range of laboratory energy we have considered, the data points number in the several hundreds and consist primarily of differential cross sections. The $\chi^{2}$ values at NLO and N3LO relative to the $p p$ data are generally significantly better than those relative to $n p$ data, except again at N3LO for model d, for which this trend is reversed (it worth reiterating here that the fits are optimized by minimizing the $\chi^{2}$ relative to the $n p$ and $p p$ data).

TABLE III. Values of the $\chi^{2} /$ datum at LO, NLO, and N3LO. The $\chi^{2} /$ datum values reported at LO over the laboratory-energy range $T_{\text {lab }}=0-1 \mathrm{MeV}$ are obtained with the LECs of Table II. The NLO (N3LO) fits are performed over the range $T_{\text {lab }}=0-15(0-25)$ MeV; $N_{n p}, N_{p p}$, and $N$ denote, respectively, the total number of $n p, p p$, and $n p+p p$ data, including observables and normalizations. The NLO and N3LO fits are carried out by enforcing that the deuteron binding energy be reproduced exactly and are optimized by minimizing the $\chi^{2}$ corresponding to the total number of $n p+p p$ data.

\begin{tabular}{|c|c|c|c|c|c|c|c|c|}
\hline Model & Order & $T_{\text {lab }}(\mathrm{MeV})$ & $N_{n p}$ & $\chi^{2}(n p) /$ datum & $N_{p p}$ & $\chi^{2}(p p) /$ datum & $N$ & $\chi^{2} /$ datum \\
\hline \multirow[t]{2}{*}{$\mathrm{a}$} & LO & $0-1$ & 91 & 5.54 & 157 & & 248 & \\
\hline & NLO & $0-15$ & 381 & 1.83 & 394 & 1.53 & 776 & 1.67 \\
\hline \multirow[t]{2}{*}{$\mathrm{b}$} & LO & $0-1$ & 91 & 37.6 & 157 & & 248 & \\
\hline & NLO & $0-15$ & 382 & 1.39 & 395 & 1.09 & 778 & 1.24 \\
\hline \multirow{2}{*}{$\mathrm{c}$} & NLO & $0-15$ & 378 & 2.34 & 392 & 1.97 & 771 & 2.15 \\
\hline & N3LO & $0-25$ & 645 & 1.83 & 453 & 1.33 & 1099 & 1.62 \\
\hline \multirow[t]{3}{*}{$\mathrm{d}$} & $\mathrm{LO}$ & $0-1$ & 91 & 41.2 & 157 & & 248 & \\
\hline & NLO & $0-15$ & 377 & 10.2 & 392 & 6.88 & 770 & 8.51 \\
\hline & N3LO & $0-25$ & 638 & 2.03 & 446 & 8.09 & 1085 & 4.52 \\
\hline
\end{tabular}


TABLE IV. Values of the $\chi^{2} /$ datum at NLO and N3LO obtained by setting $R_{0}=R_{1}=R$, namely without differentiating the range of the interactions between $T=0$ and $T=1$ pairs. Remaining notation is as in Table III. The NLO and N3LO fits are optimized by minimizing the $\chi^{2}$ corresponding to the total number of $n p+p p$ data over the same range $T_{\mathrm{lab}}=0-15 \mathrm{MeV}$. Note that the deuteron binding energy was not included in the fits.

\begin{tabular}{lcccccccc}
\hline \hline$R(\mathrm{fm})$ & Order & $T_{\text {lab }}(\mathrm{MeV})$ & $N_{n p}$ & $\chi^{2}(n p) /$ datum & $N_{p p}$ & $\chi^{2}(p p) /$ datum & $N$ & $\chi^{2} /$ datum \\
\hline 1.0 & NLO & $0-15$ & 375 & 15.5 & 390 & 8.61 & 776 & 12.0 \\
& N3LO & $0-15$ & 366 & 5.95 & 392 & 3.96 & 758 & 4.92 \\
1.5 & NLO & $0-15$ & 366 & 3.32 & 392 & 1.49 & 758 & 2.38 \\
& N3LO & $0-15$ & 369 & 1.44 & 395 & 1.64 & 764 & 1.55 \\
2.0 & NLO & $0-15$ & 367 & 2.78 & 391 & 1.46 & 758 & 2.10 \\
& N3LO & $0-15$ & 367 & 1.66 & 393 & 0.95 & 760 & 1.29 \\
2.5 & NLO & $0-15$ & 373 & 9.75 & 393 & 2.19 & 766 & 5.87 \\
& N3LO & $0-15$ & 374 & 3.48 & 392 & 1.85 & 766 & 2.64 \\
\hline \hline
\end{tabular}

We conclude this section by noting that in an earlier exploratory phase of the present work, we considered interactions regularized by a single cutoff function, namely without differentiating between pairs in isospin $T=0$ and 1 . This is equivalent to setting $R_{0}=R_{1}=R$, and

$$
C(r)=\frac{1}{\pi^{3 / 2} R^{3}} e^{-(r / R)^{2}} .
$$

Both NLO and N3LO interactions were fitted to the database over the energy range $0-15 \mathrm{MeV}$ (although the deuteron binding energy was not included in the fits), and with cutoff $R$ varying between 1.0 and $2.5 \mathrm{fm}$; see Table IV. We found the $\chi^{2}$ corresponding to the $n p$ data fits to be rather large when the cutoff $R$ was taken either too small $R \lesssim 1.0 \mathrm{fm}$ or too large $R \gtrsim 2.5 \mathrm{fm}$. Moreover, the deuteron binding energy was generally poorly reproduced at both NLO and N3LO; for example, with $R=2.5 \mathrm{fm}$ it was calculated to be 1.243 (1.312) $\mathrm{MeV}$ at NLO (N3LO). This led us to (i) introduce two cutoffs differentiating between $T=0$ and 1 pairs in order to allow for different ranges in these channel interactions, (ii) restrict the variability of the $R_{0}$ cutoff between 1.5 and $2.3 \mathrm{fm}$, in order to improve the $\chi^{2}$, and (iii) include in the fits the deuteron binding energy which, because of the small experimental error associated with it, puts a very tight constraint on the $\chi^{2}$.

\section{A. Deuteron properties, effective range parameters, and phase shifts}

Deuteron properties obtained at NLO and N3LO are reported in Table $\mathrm{V}$ and compared to available experimental values. The binding energy $B_{d}$ is fitted exactly and includes the contributions (about $20 \mathrm{keV}$ ) of electromagnetic interactions, among which the largest is that due to the magnetic moment term. The asymptotic $D / S$ ratio $\eta$ is reasonably close to experiment for models $\mathrm{a}, \mathrm{b}$, and $\mathrm{d}$ but is significantly overpredicted and underpredicted in models $\mathrm{c}$ and o, respectively.

Deuteron waves at LO, NLO, and N3LO are shown in Fig. 1. They are compared to the $S$ - and $D$ wave obtained with the AV18 [15] for reference. Note that the tensor term in the $n$ - $p$ magnetic-moment interaction induces at LO tiny $D$ waves, which are not displayed in Fig. 1. The NLO and N3LO $D$ waves in all models are smaller than the AV18 $D$ wave and are pushed out relative to it. By contrast, the LO, NLO, and N3LO $S$ waves at short range are significantly larger than the AV18 $S$ wave, reflecting the absence of a repulsive core in the contact interactions.

The effective range parameters at LO, NLO, and N3LO are given in Tables VI, VII, and VIII, respectively, where they are compared to experimental values. The experimental value of the singlet $n p$ scattering length is reproduced exactly by design in the case of the LO models a-d. However, the LO model o has been constrained to reproduce the $n p$ effective range expansions in the singlet and triplet channels as well as the deuteron binding energy. At all orders of the power counting, the singlet and triplet $n p$, and singlet $p p$ and $n n$, scattering lengths are calculated with the inclusion of electromagnetic interactions. Without the latter, the effective-range function is simply given by $F\left(k^{2}\right)=k \cot \delta=-1 / a+r k^{2} / 2$ up to terms linear in $k^{2}$. In the presence of electromagnetic interactions, a more complicated effective-range function must be used; it is given explicitly in Appendix D of Ref. [11], along with relevant references.

TABLE V. Deuteron binding energy $B_{d}$ (in MeV), $D$-to- $S$ state ratio $\eta$, and $D$-state probability $\left(P_{D}\right)$ obtained at NLO and N3LO; the experimental values [45-48] are, respectively, $B_{d}=2.224575(9) \mathrm{MeV}$ and $\eta=0.0256(4)$. The superscript * indicates that the corresponding observable is fitted.

\begin{tabular}{|c|c|c|c|c|c|c|c|c|c|c|}
\hline \multirow[b]{2}{*}{ Model } & \multicolumn{2}{|c|}{$\mathrm{a}$} & \multicolumn{2}{|c|}{$\mathrm{b}$} & \multicolumn{2}{|c|}{$\mathrm{c}$} & \multicolumn{2}{|c|}{$\mathrm{d}$} & \multicolumn{2}{|c|}{ o } \\
\hline & NLO & N3LO & NLO & N3LO & NLO & N3LO & NLO & N3LO & NLO & N3LO \\
\hline$\eta$ & 0.0233 & 0.0235 & 0.0237 & 0.0238 & 0.0373 & 0.0351 & 0.0231 & 0.0226 & 0.0169 & 0.0170 \\
\hline$P_{D}(\%)$ & 2.93 & 2.96 & 2.24 & 2.30 & 4.11 & 4.39 & 1.20 & 1.84 & 2.04 & 1.93 \\
\hline
\end{tabular}



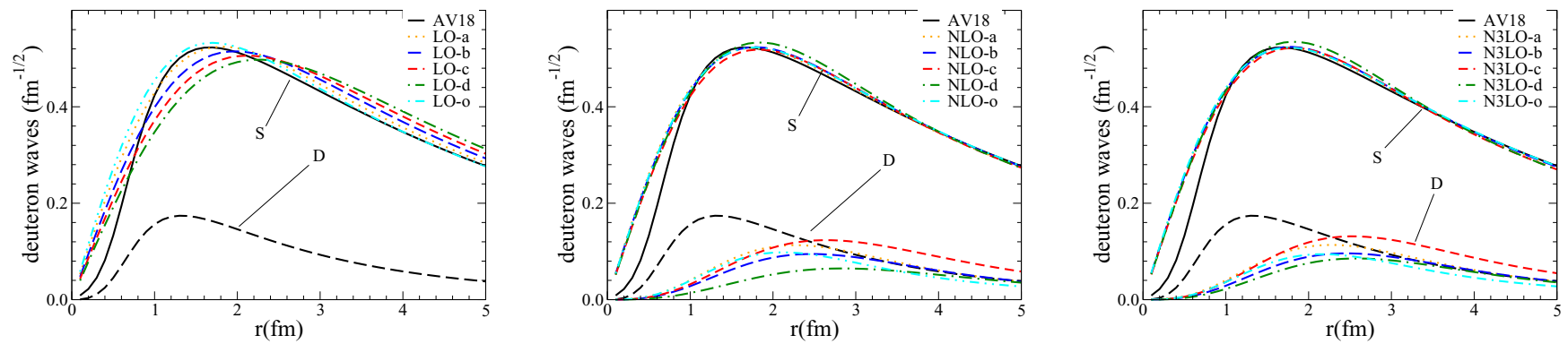

FIG. 1. The deuteron $S$-wave radial functions at LO (left panel), corresponding to the LECs of Table II, and deuteron $S$ - and $D$-wave radial functions at NLO (middle panel) and N3LO (right panel), corresponding to the best fits of Table III, are compared to those of the AV18. Note that at LO the tensor term from the $n p$ magnetic-moment interaction induces tiny $D$-wave components, which are not shown.

TABLE VI. The singlet and triplet $n p$, and singlet $p p$ and $n n$, scattering lengths and effective radii obtained at LO; experimental values are from Refs. [16,49-52]. The superscript* indicates the corresponding observable is fitted.

\begin{tabular}{|c|c|c|c|c|c|c|}
\hline & Experiment & $\mathrm{a}$ & $\mathrm{b}$ & $\mathrm{c}$ & $\mathrm{d}$ & o \\
\hline${ }^{1} a_{p p}$ & $\begin{array}{l}-7.8063(26) \\
-7.8016(29)\end{array}$ & -8.1234 & -8.8643 & -9.5462 & -10.1886 & -8.6207 \\
\hline${ }^{1} a_{n n}$ & $-18.90(40)$ & -22.13 & -22.68 & -23.01 & -23.21 & -22.53 \\
\hline${ }^{1} r_{n n}$ & $2.75(11)$ & 2.26 & 3.05 & 3.87 & 4.71 & 2.78 \\
\hline${ }^{1} r_{n p}$ & $2.77(5)$ & 2.25 & 3.04 & 2.65 & 4.69 & $2.77^{*}$ \\
\hline${ }^{3} a_{n p}$ & $5.419(7)$ & 5.515 & 5.650 & 5.783 & 5.913 & $5.410^{*}$ \\
\hline${ }^{3} r_{n p}$ & $1.753(8)$ & 1.89 & 2.06 & 2.21 & 2.36 & $1.757^{*}$ \\
\hline
\end{tabular}

TABLE VII. Same as in Table VI but at NLO.

\begin{tabular}{lccccc}
\hline \hline & Experiment & $\mathrm{a}$ & $\mathrm{b}$ & $\mathrm{c}$ & $\mathrm{d}$ \\
\hline${ }^{1} a_{p p}$ & $-7.8063(26)$ & -7.7489 & -7.7557 & -7.7463 & -7.7119 \\
& $-7.8016(29)$ & & & -7.7570 \\
${ }^{1} r_{p p}$ & $2.794(14)$ & 2.649 & 2.676 & 2.622 & 2.439 \\
& $2.773(14)$ & & & -16.90 & -16.45 \\
${ }^{1} a_{n n}$ & $-18.90(40)$ & -17.19 & -17.21 & 2.79 & -17.23 \\
${ }^{1} r_{n n}$ & $2.75(11)$ & 2.78 & 2.80 & -23.740 \\
${ }^{1} a_{n p}$ & $-23.740(20)$ & -23.765 & -23.740 & 2.78 & -23.738 \\
${ }^{1} r_{n p}$ & $2.77(5)$ & 2.71 & 2.75 & 5.418 & 2.78 \\
${ }^{3} a_{n p}$ & $5.419(7)$ & 5.392 & 5.424 & 1.831 & 5.415 \\
${ }^{3} r_{n p}$ & $1.753(8)$ & 1.746 & 1.796 & 5.426 \\
\hline \hline
\end{tabular}

TABLE VIII. Same as in Table VI but at N3LO.

\begin{tabular}{lccccc}
\hline \hline & Experiment & $\mathrm{a}$ & $\mathrm{b}$ & $\mathrm{c}$ & $\mathrm{d}$ \\
\hline${ }^{1} a_{p p}$ & $-7.8063(26)$ & -7.7539 & -7.7634 & -7.7554 & -7.7730 \\
& $-7.8016(29)$ & & & & -7.7590 \\
${ }^{1} r_{p p}$ & $2.794(14)$ & 2.669 & 2.709 & 2.674 & 2.744 \\
& $2.773(14)$ & & & -17.13 & -16.72 \\
${ }^{1} a_{n n}$ & $-18.90(40)$ & -17.15 & -17.22 & 2.80 & -17.23 \\
${ }^{1} r_{n n}$ & $2.75(11)$ & 2.80 & 2.83 & -23.780 & -23.794 \\
${ }^{1} a_{n p}$ & $-23.740(20)$ & -23.760 & -23.745 & 2.49 & 2.15 \\
${ }^{1} r_{n p}$ & $2.77(5)$ & 2.68 & 2.60 & 5.366 & 2.739 \\
${ }^{3} a_{n p}$ & $5.419(7)$ & 5.397 & 5.415 & 1.769 & 5.422 \\
${ }^{3} r_{n p}$ & $1.753(8)$ & 1.754 & 1.784 & & 1.776 \\
\hline \hline
\end{tabular}



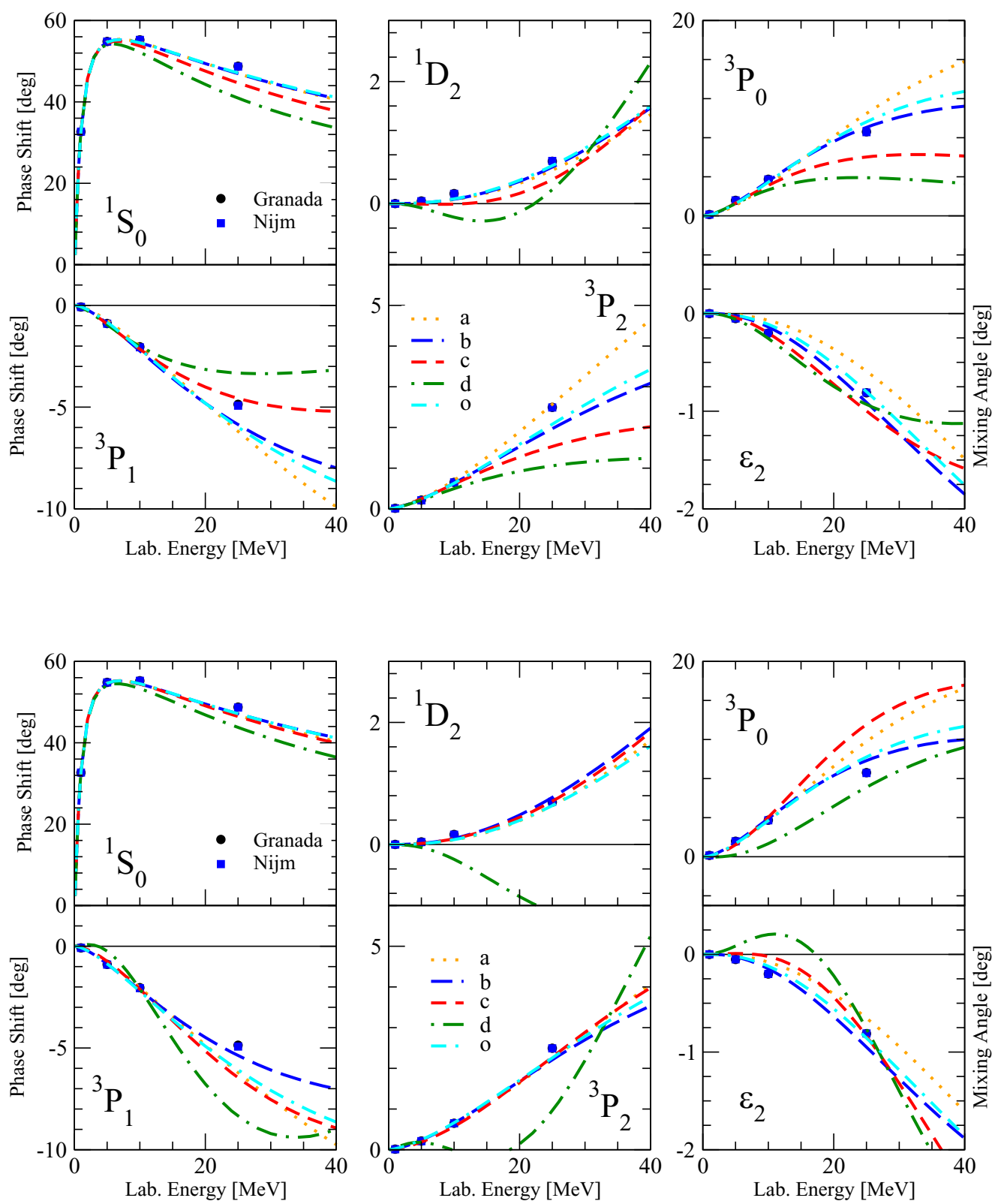

FIG. 2. Phase shifts in $p p$ channels at NLO (top panel) and N3LO (bottom panel) corresponding to the best fits of Table III are compared to the results of the Nijmegen and Granada partial-wave analyses.

The predicted scattering lengths at NLO and N3LO are typically within $1 \%$ of the experimental values for all models considered. However, the effective radii display more variability from model to model, but are all reasonably close to experiment.

The $p p$ and (isovector and isoscalar) $n p S$-, $P$-, and $D$-wave phase shifts obtained with the NLO and N3LO interaction models up to a laboratory energy of $40 \mathrm{MeV}$ are displayed in Figs. 2-4 and are compared to partial-wave analyses (PWAs) by the Nijmegen [53], Granada [41-43], and Gross-Stadler [54] groups. The Gross-Stadler PWA is limited to $n p$ data only. The $p p$ phases are relative to electromagnetic functions [11], while the $n p$ ones are relative to spherical Bessel functions. Except for the $S$-wave phase shifts, there is a rather large spread in the $P$ - and $D$-wave phase shifts and mixing angles among the different models. This spread does not appear to be reduced in going from NLO to N3LO, although it is worthwhile reiterating here that the fits to the database were restricted to a rather low upper limit in the energy range and that in such a range the data, which consist primarily of differential cross sections, are not very sensitive to higher order partial waves.

\section{BINDING ENERGIES OF LIGHT AND MEDIUM-WEIGHT NUCLEI}

In this section, we report the results for the binding energies of ${ }^{3} \mathrm{H},{ }^{3} \mathrm{He},{ }^{4} \mathrm{He},{ }^{6} \mathrm{He},{ }^{6} \mathrm{Li},{ }^{16} \mathrm{O},{ }^{40} \mathrm{Ca},{ }^{48} \mathrm{Ca}$, and ${ }^{90} \mathrm{Zr}$. The calculations are carried out with $2 \mathrm{~N}$ interactions up to N3LO in the $A=3-6$ systems, up to NLO in ${ }^{16} \mathrm{O}$, and at 

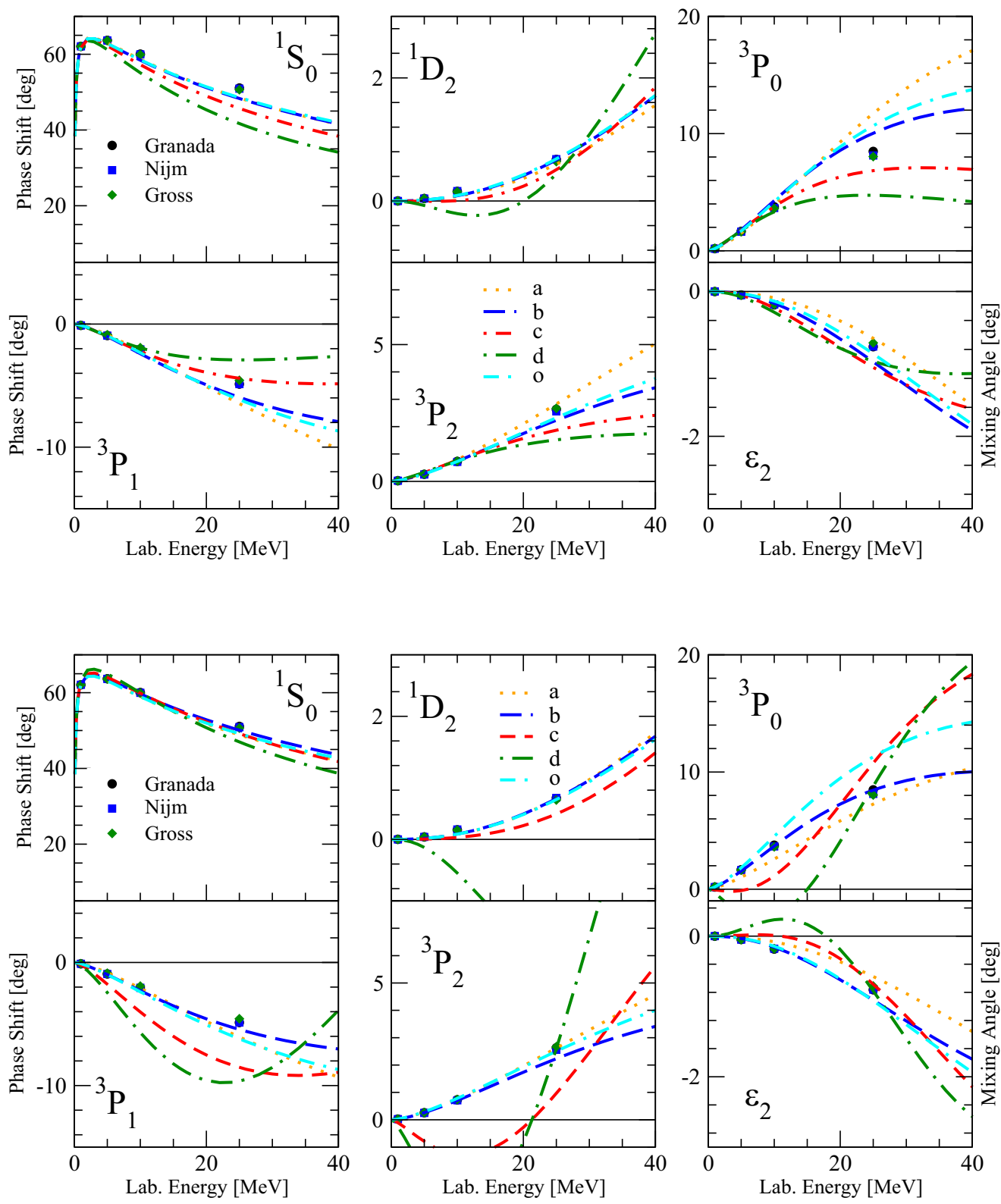

FIG. 3. Phase shifts in isovector $n p$ channels at NLO (top panel) and N3LO (bottom panel) corresponding to the best fits of Table III are compared to the results of the Nijmegen, Granada, and Gross partial-wave analyses.

LO only for the heavier nuclei with $A \geqslant 40$, and make use of hyperspherical-harmonics (HH) methods in $A \leqslant 6$ and auxiliary-field diffusion Monte Carlo (AFDMC) methods for $A \geqslant 16$; see below. Of course, a consistent study of nuclei must retain the complete interaction at the different orders. In the present work, which deals primarily with the construction of $2 N$ interactions, we include the three-nucleon $(3 N)$ contact interaction at $\mathrm{LO}$ only and postpone the study of higher order $3 N$ terms [55] to a subsequent work (a preliminary study of these higher order terms can be found in Ref. [56]). At LO, we consider

$$
V_{\mathrm{LO}}=c_{E} \frac{f_{\pi}^{4}}{\Lambda_{\chi}} \frac{(\hbar c)^{6}}{\pi^{3} R_{3}^{6}} \sum_{\text {cyclic } i j k} e^{-\left(r_{i j}^{2}+r_{j k}^{2}\right) / R_{3}^{2}}
$$

where $\Lambda_{\chi}=1 \mathrm{GeV}$ is the breaking scale of the theory and $f_{\pi}=92.4 \mathrm{MeV}$ is the pion decay constant. The LEC $c_{E}$ can be determined from a single three-nucleon data point for different choices of the range $R_{3}$. Examples of this procedure can be found in Refs. $[24,57,58]$, where correlations between the three-, four-, six-nucleon systems and nuclear matter have been analyzed. Here, for each $2 N$ model, we fix $c_{E}$ to reproduce $B\left({ }^{3} \mathrm{H}\right)=8.475 \mathrm{MeV}$, for different choices of the cutoff $R_{3}$. The values so obtained are listed in Table IX. They are generally found to be natural, at least when the cutoff $R_{3}$ is in the range $1.0 \lesssim R_{3} \lesssim 2.0 \mathrm{fm}$ for models $\mathrm{a}, \mathrm{b}$, and $\mathrm{o}$.

\section{A. Binding energies of $A=3,4$, and 6 nuclei with $\mathrm{HH}$ methods}

The ${ }^{3} \mathrm{H},{ }^{3} \mathrm{He},{ }^{4} \mathrm{He},{ }^{6} \mathrm{Li}$, and ${ }^{6} \mathrm{He}$ binding energies obtained with the different $2 N$ contact interactions are reported in 

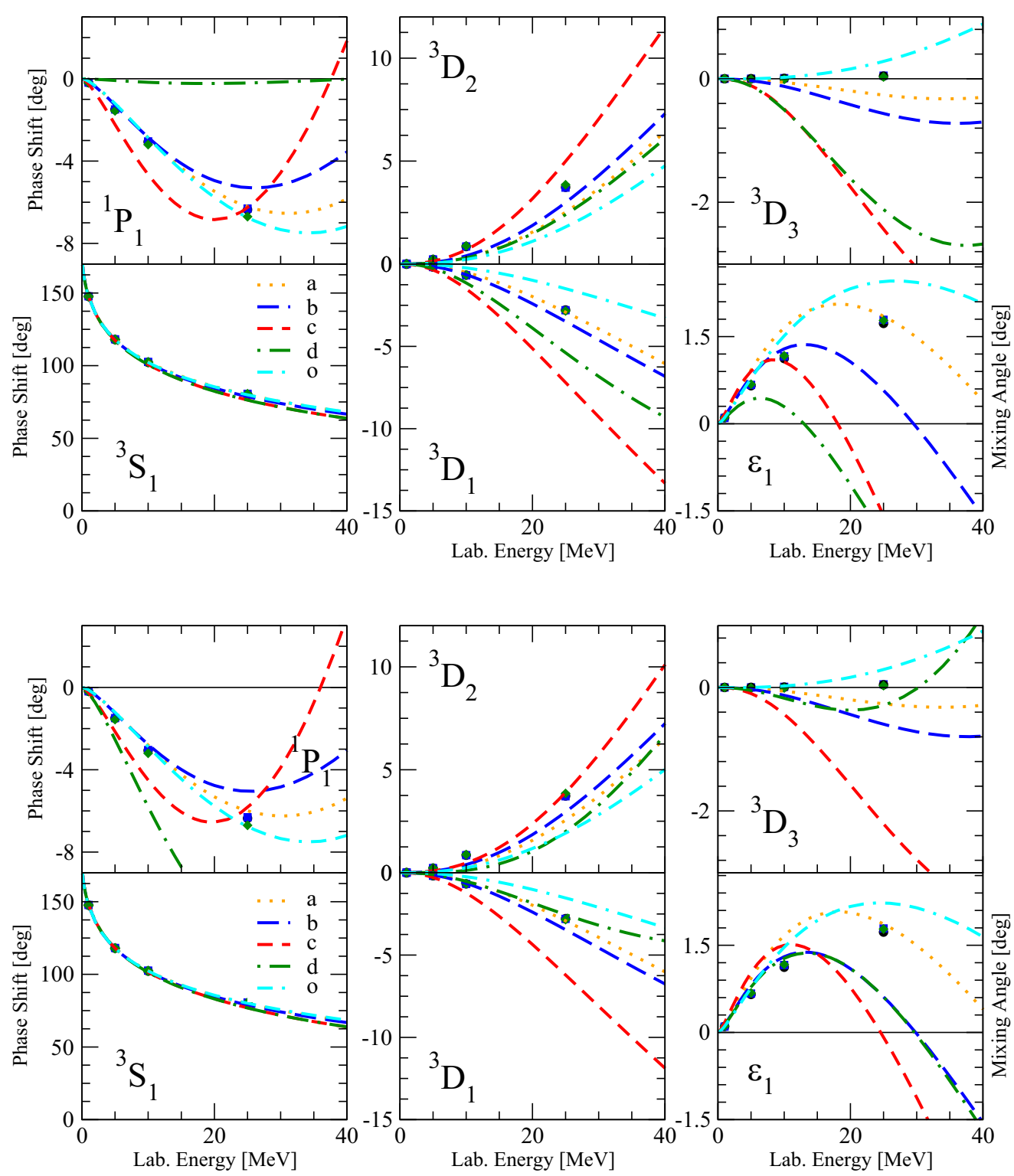

FIG. 4. Phase shifts in isoscalar $n p$ channels at NLO (top panel) and N3LO (bottom panel) corresponding to the best fits of Table III are compared to the results of the Nijmegen, Granada, and Gross partial-wave analyses.

Table X. As already noted, the calculations have been carried out with the $\mathrm{HH}$ method, as described in the recent reviews $[59,60]$ (and references therein). These binding energies are expected to be accurate at the level of 1 and $10 \mathrm{keV}$ for the three- and four-nucleon systems, respectively. For the sixnucleon system, the $\mathrm{HH}$ basis is largely degenerate, requiring detailed studies. Accordingly, the $\mathrm{HH}$ states are partitioned in different "classes of convergence" and within each of these an extrapolation is made to estimate the missing energy. The estimates for all classes of convergence are then added up to obtain the total extrapolated value for the binding energy. A complete discussion of these aspects-in particular, the definition of classes of convergence, and the extrapolation in each of these classes - can be found in Ref. [61] for ${ }^{6} \mathrm{Li}$ and in Ref. [62] for ${ }^{6} \mathrm{He}$. The errors on the extrapolated energies are in general larger for ${ }^{6} \mathrm{He}$ because of the loosely bound structure and the slower convergence as compared to ${ }^{6} \mathrm{Li}$.
We find that at LO there is a large spread in the results, reflecting a large dependence on the cutoffs. The three-nucleon binding energies vary by more than $4 \mathrm{MeV}$, whereas the spread in the four- and six-nucleon binding energies exceeds $20 \mathrm{MeV}$. This large variation as a function of the cutoffs is related to the Thomas collapse phenomenon [63]: As the range of the interaction is reduced, these systems tend to become more bound. This is especially apparent in the limiting case in which the interaction is of zero range, corresponding to the limiting case of the LO interaction. As is apparent from Table X, the dependence on the cutoffs is much less drastic for the NLO and N3LO interactions. However, the ${ }^{6} \mathrm{Li}$ and ${ }^{6} \mathrm{He}$ results show a peculiar behavior, in that at N3LO the spread is relatively small, about $3 \mathrm{MeV}$, for the $\mathrm{a}, \mathrm{b}, \mathrm{c}$, and o models; on the other hand, model d seems to be an outlier and yields large binding energies, when compared to those of the other models. Lastly, it should be noted that ${ }^{6} \mathrm{He}$ is 
TABLE IX. Values obtained at LO, NLO, and N3LO for the LEC $c_{E}$ in the $3 N$ contact interaction, corresponding to cutoffs $R_{3}=1.0$, 1.5, 2.0, and $2.5 \mathrm{fm}$. Each combination is constrained to reproduce the ${ }^{3} \mathrm{H}$ binding energy in $\mathrm{HH}$ calculations. Parts (A) and (B) report the $c_{E}$ values obtained by either ignoring (A) or retaining (B) the full $v^{E M}$ in the ${ }^{3} \mathrm{H}$ calculations.

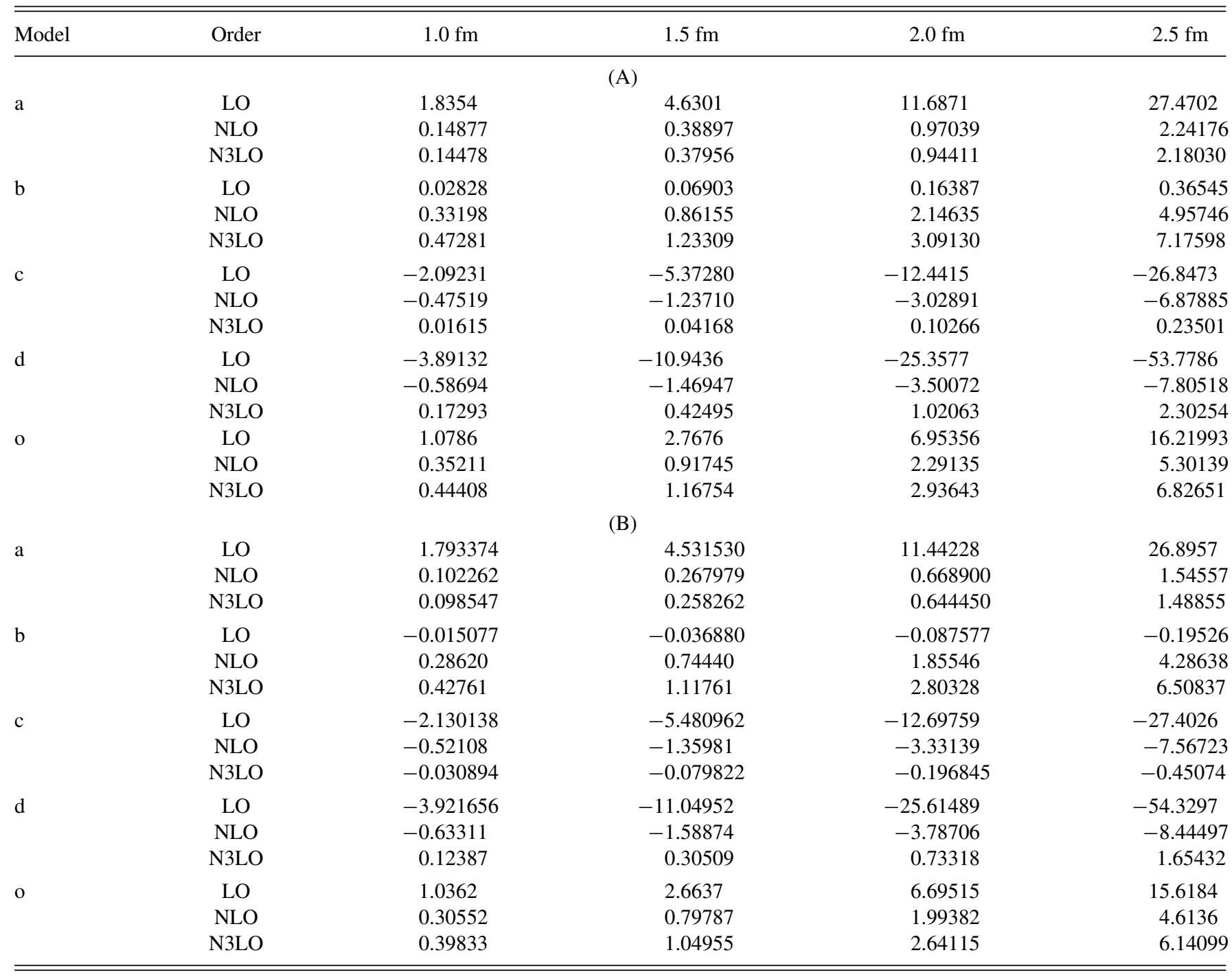

found to be bound with all models, except with model $\mathrm{c}$ at NLO.

Next, we include the $3 N$ contact interaction discussed earlier in the binding energy calculations of the $A=3$ and 4 nuclei. The results are summarized in Fig. 5. The left, center, and right panels present the binding energies obtained with the $2 N$ contact interactions at LO, NLO, and N3LO (each including the full electromagnetic interaction and hence each in combination with the LO $3 N$ contact interaction corresponding to set $\mathrm{B}$ of $c_{E}$ values), whereas the different symbols in each panel correspond to the five possible choices of $2 \mathrm{~N}$ cutoffs. The lower and upper subpanels show the $B\left({ }^{3} \mathrm{He}\right)$ and $B\left({ }^{4} \mathrm{He}\right)$ results (note the energy scales). The two dashed lines indicate the experimental values, $B\left({ }^{3} \mathrm{He}\right)=7.72 \mathrm{MeV}$ and $B\left({ }^{4} \mathrm{He}\right)=28.3 \mathrm{MeV}$. The constraint $B\left({ }^{3} \mathrm{H}\right)=8.475 \mathrm{MeV}$ is verified by all models by construction. The figure shows that at $\mathrm{LO}$ a fine tuning of the cutoffs in the $2 N$ interaction could be used to reproduce $B\left({ }^{4} \mathrm{He}\right)$, since models a and b are just below and above the experimental value; such fine-tuning is in fact achieved with model o. Increasing the order of the expansion, at NLO and N3LO, leads to much more stable results, clustering around $7.78 \mathrm{MeV}$ for $B\left({ }^{3} \mathrm{He}\right)$ and around $30 \mathrm{MeV}$ for $B\left({ }^{4} \mathrm{He}\right)$. We expect these binding energies to be correctly reproduced by including higher order terms in the $3 N$ interaction.

\section{B. ${ }^{16}$ O nucleus with AFDMC methods}

The auxiliary-field diffusion Monte Carlo (AFDMC) method [64] is used to study nuclei with $A>6$ nucleonssee Ref. [65] for a recent review (see also Ref. [66] for AFDMC applications with NLO $\chi$ EFT interactions). The AFDMC method uses imaginary-time projection techniques to filter out the ground state of the system starting from a suitable trial wave function, $\left|\Psi_{0}\right\rangle=e^{-\left(H-E_{0}\right) \tau}\left|\Psi_{T}\right\rangle$, and exhibits a favorable polynomial scaling with the number of nucleons, which is made possible by the use of a single-particle spin-isospin basis. This representation is preserved during 
TABLE X. Binding energies (in MeV) corresponding to the $2 N$ contact interaction models a-d and o, obtained at LO, NLO, and N3LO with the $\mathrm{HH}$ method for nuclei with mass numbers $A=3,4$, and 6 ; the numbers in parentheses for $A=6$ are estimates of extrapolation errors (see text). The experimental values are 8.48, 7.72, 28.3, 32.0, and $29.3 \mathrm{MeV}$ for, respectively, ${ }^{3} \mathrm{H},{ }^{3} \mathrm{He},{ }^{4} \mathrm{He},{ }^{6} \mathrm{Li}$, and ${ }^{6} \mathrm{He}$.

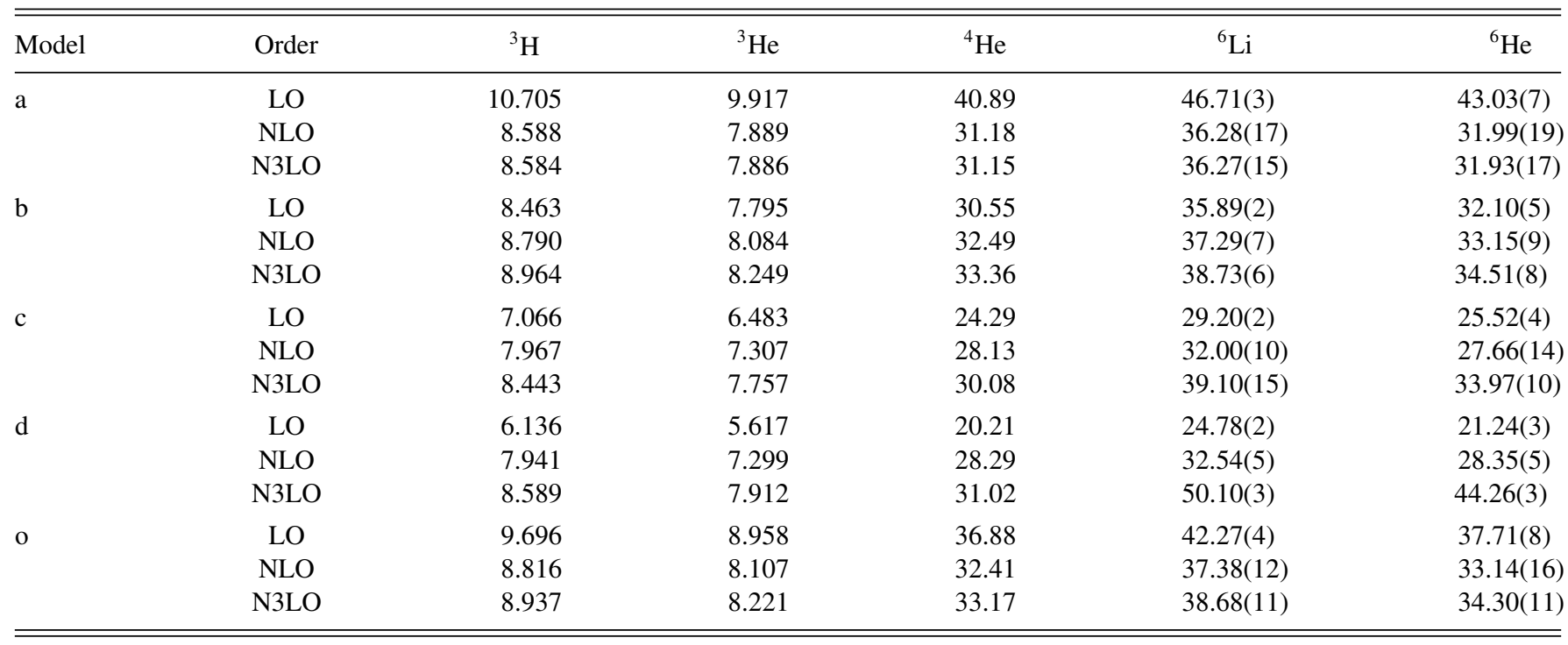

the imaginary-time evolution by using Hubbard-Stratonovich transformations to linearize the quadratic spin-isospin operators entering the short-time propagator. Applying these transformation to treat the isospin-dependent spin-orbit term, implicit in the $v_{\mathrm{NLO}}^{\mathrm{CI}}$ interaction, involves nontrivial difficulties. To circumvent them, we perform the imaginary-time

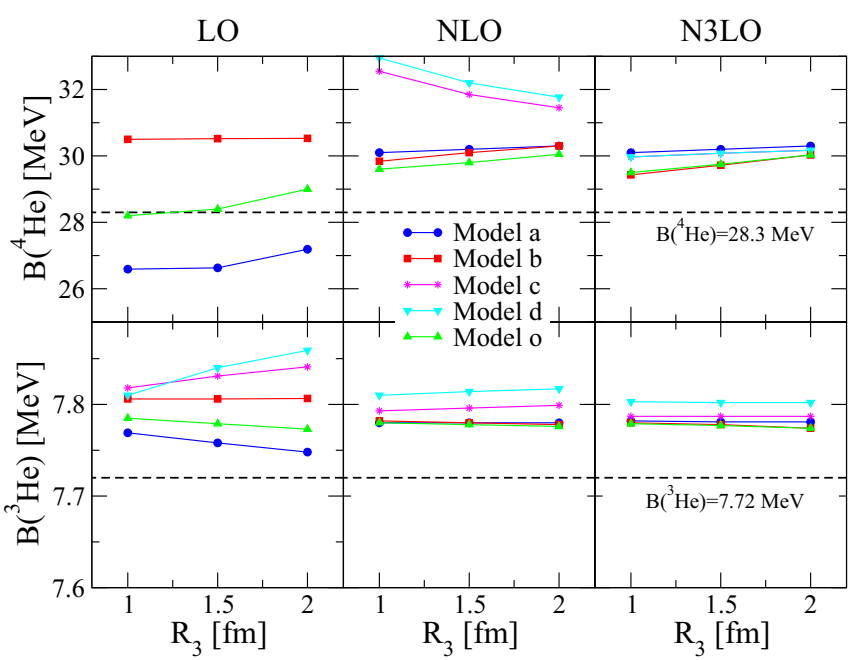

FIG. 5. Binding energies of ${ }^{3} \mathrm{He}$ (lower subpanels) and ${ }^{4} \mathrm{He}$ (upper subpanels) with the inclusion of the LO $3 N$ contact interaction corresponding to set $\mathrm{B}$, determined by fitting the ${ }^{3} \mathrm{H}$ binding energy, as a function of the cutoff $R_{3}$. The left, center, and right panels refer to the LO, NLO, and N3LO $2 N$ contact interactions (each in combination with the LO $3 \mathrm{~N}$ contact interaction), while circles, squares, stars, triangles down, and triangles up correspond to models a-d and o, respectively. The lines are only drawn to guide the eye. For ${ }^{4} \mathrm{He}$, the LO binding energies obtained with models $\mathrm{c}$ and $\mathrm{d}$ are out of scale (too large) and are not shown. The dashed lines indicate the experimental values; note the different energy scales adopted for ${ }^{3} \mathrm{He}$ and ${ }^{4} \mathrm{He}$. propagation with a modified interaction, as described below. The $v_{\mathrm{NLO}}^{\mathrm{CI}}$ interactions reads

$$
v_{\mathrm{NLO}}^{\mathrm{CI}}=\sum_{l=1}^{6} v^{l}(r) O_{12}^{l}+\left[v^{b}(r)+v^{b \tau}(r) \boldsymbol{\tau}_{1} \cdot \boldsymbol{\tau}_{2}\right] \mathbf{L} \cdot \mathbf{S}
$$

where, referring to Appendix A, the radial functions of the spin-orbit components are defined as

$$
\begin{aligned}
& v^{b}(r)=-\frac{C_{7}}{r} \frac{3 C_{1}^{(1)}(r)+C_{0}^{(1)}(r)}{4}, \\
& v^{b \tau}(r)=-\frac{C_{7}}{r} \frac{C_{1}^{(1)}(r)-C_{0}^{(1)}(r)}{4},
\end{aligned}
$$

with $C_{T}^{(1)}(r)$ denoting the derivative of the Gaussian cutoff in isospin channel $T$. The isospin dependence of the spin-orbit term comes on account of the fact that $C_{0}^{(1)}(r)$ and $C_{1}^{(1)}(r)$ have different ranges. The modified NLO interaction is defined as

$$
v_{\mathrm{NLO}}^{\mathrm{CI} \prime}(\alpha)=\sum_{1=1}^{6} v^{l}(r) O_{12}^{l}+\left[v^{b}(r)+\alpha v^{b \tau}(r)\right] \mathbf{L} \cdot \mathbf{S} .
$$

The imaginary time propagation is performed with this modified interaction. The expectation values of both $v_{\mathrm{NLO}}^{\mathrm{CI} \prime}(\alpha)$ and the original $v_{\mathrm{NLO}}^{\mathrm{CI}}$ are then evaluated, and the parameter $\alpha$ is adjusted so as to make these expectation values the same within statistical errors. Note that a similar procedure has been adopted in Refs. $[67,68]$ to include the commutator term of the three-body chiral interaction.

The trial wave function is expressed as a product of a long-range Slater determinant of single-particle orbitals and a correlation factor, $\left|\Psi_{T}\right\rangle=F|\Phi\rangle$. Since the NLO Hamiltonian contains both tensor and spin-orbit terms, we consider a trial wave function that includes linear spin-isospin dependent 
TABLE XI. Binding energies (in MeV) of ${ }^{3} \mathrm{H}$ and ${ }^{4} \mathrm{He}$ obtained with LO and NLO $2 N$ interactions using the AFDMC method in the constrained-path approximation are compared to corresponding $\mathrm{HH}$ results. Estimated statistical errors in the AFDMC calculations are in parentheses. Note that the electromagnetic interaction only includes the Coulomb repulsion between finite-size (rather than point-like) protons.

\begin{tabular}{|c|c|c|c|c|c|}
\hline Model & Order & $B\left({ }^{3} \mathrm{H}\right)$ & $B\left({ }^{3} \mathrm{H}\right)[\mathrm{HH}]$ & $B\left({ }^{4} \mathrm{He}\right)$ & $B\left({ }^{4} \mathrm{He}\right)[\mathrm{HH}]$ \\
\hline $\mathrm{a}$ & $\begin{array}{c}\text { LO } \\
\text { NLO }\end{array}$ & $\begin{array}{r}10.75(2) \\
8.64(1)\end{array}$ & $\begin{array}{r}10.756 \\
8.639\end{array}$ & $\begin{array}{l}41.10(5) \\
31.50(2)\end{array}$ & $\begin{array}{l}41.10 \\
31.37\end{array}$ \\
\hline b & $\begin{array}{c}\text { LO } \\
\text { NLO }\end{array}$ & $\begin{array}{l}8.47(1) \\
8.82(1)\end{array}$ & $\begin{array}{l}8.498 \\
8.839\end{array}$ & $\begin{array}{l}30.71(1) \\
32.75(1)\end{array}$ & $\begin{array}{l}30.69 \\
32.68\end{array}$ \\
\hline $\mathrm{c}$ & $\begin{array}{c}\text { LO } \\
\text { NLO }\end{array}$ & $\begin{array}{l}7.07(1) \\
7.96(2)\end{array}$ & $\begin{array}{l}7.093 \\
8.013\end{array}$ & $\begin{array}{l}24.40(1) \\
28.33(2)\end{array}$ & $\begin{array}{l}24.40 \\
28.31\end{array}$ \\
\hline o & $\begin{array}{c}\text { LO } \\
\text { NLO }\end{array}$ & $\begin{array}{l}9.71(1) \\
8.85(1)\end{array}$ & $\begin{array}{l}9.744 \\
8.867\end{array}$ & $\begin{array}{l}37.08(3) \\
32.66(3)\end{array}$ & $\begin{array}{l}37.07 \\
32.60\end{array}$ \\
\hline
\end{tabular}

correlations [69]

$$
\begin{aligned}
F= & \prod_{i<j<k}\left[1+\sum_{\mathrm{cyc}} u_{3 \mathrm{~b}}\left(r_{i j}\right) u_{3 \mathrm{~b}}\left(r_{i k}\right)\right] \\
& \times\left[1+\sum_{i<j} \sum_{l=2}^{6} u^{l}\left(r_{i j}\right) O_{i j}^{l}\right] \prod_{i<j} f^{c}\left(r_{i j}\right),
\end{aligned}
$$

where the spin-isospin operators are defined in Eq. (2.9). The functions $u^{l}(r)$ are characterized by a number of variational parameters [65], which are determined by minimizing the two-body cluster contribution to the energy per particle of nuclear matter at saturation density. On the other hand, the function $u_{3 \mathrm{~b}}(r)$ associated with the correlations induced by the (LO) $3 N$ contact interaction and the function $f^{c}(r)$ are parametrized in terms of cubic splines. The variational parameters are the values of $u_{3 \mathrm{~b}}(r)$ and $f^{c}(r)$ at the grid points, plus the value of their first derivatives at $r=0$. The optimal values of the variational parameters are found employing the linear optimization method [70], which typically converges in $\approx 20$ iterations. When solving the LO Hamiltonian, which does not contain tensor or spin-orbit terms, we drop the spin-isospindependent correlations in Eq. (4.6). This simplified ansatz is consistent with that adopted in Ref. [70], and allows us to compute nuclei as large as ${ }^{90} \mathrm{Zr}$ with multiple LO Hamiltonians.

In Table XI, we report the AFDMC binding energies of ${ }^{3} \mathrm{H}$ and ${ }^{4} \mathrm{He}$ obtained in the constrained-path approximation

TABLE XII. Binding energies (in MeV) of ${ }^{4} \mathrm{He}$ and ${ }^{16} \mathrm{O}$ at LO, NLO, and N3LO obtained with selected combinations of contact $2 \mathrm{~N}+3 \mathrm{~N}$ interaction models, and corresponding to different cutoffs in the $3 \mathrm{~N}$ interaction, as reported in Table IX. Note that in these calculations we have retained in $v^{\mathrm{EM}}$ only the Coulomb interaction between protons (albeit accounting for their finite size). Consequently, we have used the $c_{E}$ values reported in part (A) of Table IX. The $A=4$ results are calculated with both the HH method and, in square brackets, the AFDMC

\begin{tabular}{|c|c|c|c|c|c|c|c|c|c|}
\hline Model & Order & $A=4$ & $A=16$ & $A=4$ & $A=16$ & $A=4$ & $A=16$ & $A=4$ & $A=16$ \\
\hline \multirow{2}{*}{$\mathrm{a}$} & NLO & 31.37 & $424.7(4)$ & $30.08[30.19(2)]$ & $260.7(8)$ & $30.20[30.31(2)]$ & $243.2(4)$ & $30.30[30.39(3)]$ & $243.6(4)$ \\
\hline & N3LO & 31.15 & & 30.08 & & 30.20 & & 30.30 & \\
\hline $\mathrm{b}$ & LO & 30.69 & $262.8(9)$ & $30.50[30.51(2)]$ & $260.5(9)$ & $30.52[30.52(2)]$ & $251.0(6)$ & $30.53[30.53(2)]$ & 249.3(8) \\
\hline \multirow[t]{3}{*}{$\mathrm{c}$} & LO & 24.39 & 206.9(8) & 47.47 & & 36.98 & & 34.67 & \\
\hline & NLO & 28.31 & $317.7(3)$ & 32.55 & & 31.85 & & 31.45 & \\
\hline & N3LO & 30.26 & & 30.13 & & 30.14 & & 30.15 & \\
\hline d & LO & 20.29 & $170.1(4)$ & 139.1 & & 49.46 & & 40.24 & \\
\hline o & N3LO & 33.17 & & 29.47 & & 29.75 & & 30.03 & \\
\hline
\end{tabular}
method in the constrained-path approximation; the latter method is used in the $A=16$ calculations. 
TABLE XIII. Binding energies (in MeV) of light- and medium-mass nuclei at LO predicted by selected combinations of contact $2 N+3 N$ interaction models, corresponding to different cutoffs in the $3 N$ interaction as reported in Table IX. The calculations retain in $v^{\mathrm{EM}}$ only the Coulomb repulsion between finite-size (rather than pointlike) protons. Consequently, the $c_{E}$ values reported in part (A) of Table IX have been used for the (LO) $3 N$ interaction. The $A=4-6$ and $A \geqslant 16$ results are obtained, respectively, with the HH method and AFDMC method in the constrained-path approximation.

\begin{tabular}{|c|c|c|c|c|c|c|c|c|c|c|}
\hline Nucleus & \multicolumn{3}{|c|}{$R_{3}=1.0 \mathrm{fm}$} & \multicolumn{3}{|c|}{$R_{3}=1.5 \mathrm{fm}$} & \multicolumn{3}{|c|}{$R_{3}=2.0 \mathrm{fm}$} & Exp. \\
\hline${ }^{4} \mathrm{He}$ & 26.59 & 30.50 & 28.18 & 26.63 & 30.52 & 28.49 & 27.19 & 30.53 & 29.00 & 28.30 \\
\hline${ }^{6} \mathrm{Li}$ & $28.55(2)$ & $35.62(1)$ & $30.77(1)$ & $26.70(3)$ & $35.59(1)$ & $29.51(1)$ & $25.07(5)$ & $35.57(1)$ & $28.28(1)$ & 31.99 \\
\hline${ }^{6} \mathrm{He}$ & $25.73(2)$ & $31.89(3)$ & $27.26(3)$ & $23.96(5)$ & $31.87(3)$ & $26.20(6)$ & $22.46(9)$ & $31.85(3)$ & $25.22(9)$ & 29.27 \\
\hline${ }^{16} \mathrm{O}$ & $111.6(3)$ & $260.5(9)$ & $133.4(4)$ & $76.5(8)$ & $251.0(6)$ & $96.6(4)$ & 69.1(9) & 249.3(8) & $69.7(5)$ & 127.62 \\
\hline${ }^{90} \mathrm{Zr}$ & $654(2)$ & $6511(9)$ & $937(6)$ & 216.9(8) & $6866(9)$ & $392(2)$ & & $6363(8)$ & & 783.90 \\
\hline
\end{tabular}

using the linearized spin-isospin correlations of Eq. (4.6) and compare them with the $\mathrm{HH}$ results. Since in the AFDMC the electromagnetic interaction only includes the Coulomb repulsion between finite-size (rather than point-like) protons, for a more meaningful comparison, the $\mathrm{HH}$ binding energies are also obtained with this approximation; hence these energies are slightly different from those of Table $\mathrm{X}$ which retain the full electromagnetic interaction. The AFDMC and $\mathrm{HH}$ results for ${ }^{3} \mathrm{H}$ are in excellent agreement with each other: The largest discrepancy between the two methods is $\approx 0.05 \mathrm{MeV}$ for model $\mathrm{c}$ at NLO; differences between AFDMC and $\mathrm{HH}$ results are much smaller for all the other models. A similar trend is observed for the ${ }^{4} \mathrm{He}$ nucleus. The AFDMC and $\mathrm{HH}$ energies are quite close; the largest discrepancy, $\approx 0.13 \mathrm{MeV}$, is again observed for model a at NLO and is smaller for all other models we analyzed. Some of these differences can be ascribed to a combination of the constrained-path approximation employed in the AFDMC, the approximate treatment of the isospin-dependent spin-orbit term of the interaction, and the convergence of the $\mathrm{HH}$ basis expansion.

The binding energies of ${ }^{4} \mathrm{He}$ and ${ }^{16} \mathrm{O}$ at LO, NLO, and $\mathrm{N} 3 \mathrm{LO}$ for selected $2 \mathrm{~N}$ models and including the $3 \mathrm{~N}$ interaction are listed in Table XII. The agreement between $\mathrm{HH}$ and AFDMC calculations of ${ }^{4} \mathrm{He}$ - the latter reported in square brackets-remains excellent even when the $3 N$ interaction is included in the Hamiltonian. We note that neglecting the $3 \mathrm{~N}$ interaction always yields too large a binding energy in ${ }^{16} \mathrm{O}$, even when the ${ }^{4} \mathrm{He}$ is underbound. On the other hand, fixing the $3 N$ interaction to reproduce the ${ }^{3} \mathrm{H}$ binding energy leads to a sizable cutoff dependence of our results, regardless of the $2 N$ interaction model considered. In general, a larger cutoff $R_{3}$ corresponds to a lesser bound ${ }^{16} \mathrm{O}$, as the repulsive term becomes long-ranged and affects triplets of nucleons belonging to different $\alpha$-like clusters. In this regard, we observe that in some cases the AFDMC binding energies of ${ }^{16} \mathrm{O}$ are smaller than four times that of ${ }^{4} \mathrm{He}$. Although a fully clusterized wave function can be obtained as done in Ref. [70], in this work we use confining single-particle orbitals that effectively prevent the nucleons from diffusing far from the center of mass of the system. Finally, we refrain from carrying out AFDMC calculations of ${ }^{16} \mathrm{O}$ for models $\mathrm{c}$ and $\mathrm{d}$, since for these the LEC $c_{E}$ is negative and the corresponding $3 N$ interaction would therefore lead to large additional binding for the already overbound results predicted by the $2 \mathrm{~N}$ models alone.

In Table XIII, we list the binding energies of selected lightand medium-mass nuclei at $\mathrm{LO}$ computed using the $\mathrm{HH}$ and AFDMC methods. We observe a similar trend as in Table XII, with a sizable dependence of the results on the cutoff $R_{3}$. Overbinding or underbinding in ${ }^{16} \mathrm{O}$ carries over in heavier nuclei. On a positive note, models a and o for $R_{3}=1.0 \mathrm{fm}$ provide a satisfactory description of ${ }^{16} \mathrm{O}$ and are also able to reproduce fairly well the binding energies of heavier systems. It would be interesting to fit $c_{E}$ by reproducing ${ }^{16} \mathrm{O}$, as done, for instance, in Ref. [71], and study the behavior of such a Hamiltonian in lighter and heavier nuclei.

\section{SUMMARY AND CONCLUSIONS}

The present work represents the first phase in a program we envision, aimed at establishing whether the energy spectra of, and electroweak transitions between, low-lying states of nuclei can be understood on the basis of nuclear interactions and electroweak currents, derived in an EFT formulation where pion degrees of freedom have been integrated out. Specifically, this first phase has dealt with (i) the construction of $2 \mathrm{~N}$ contact interactions at LO, NLO, and N3LO that are local in configuration space and therefore suitable for implementation in quantum Monte Carlo calculations; (ii) the determination of a $3 N$ contact interaction at LO with the single LEC entering at this order fixed to reproduce the tritium binding energy in essentially exact $\mathrm{HH}$ calculations; (iii) the extension of the AFDMC method, so as to be capable of handling approximately but reliably tensor and spin-orbit components (with and without isospin dependence) in the $2 N$ interactions; (iv) a fairly complete study (albeit not a fully consistent one from a power counting perspective) of the ground-state binding energies of light nuclei with mass number up to $A=16$ with Hamiltonians based on $2 N$ interactions of increasing order but a $3 N$ interaction included only at LO; (v) an initial set of AFDMC calculations of the binding energies of nuclei with $A \geqslant 40$ based on Hamiltonians including the contact $2 N$ and $3 N$ interactions at LO only.

The fits to the $2 N$ scattering database (including the deuteron binding energy) have been restricted up to laboratory 
energies of $15 \mathrm{MeV}$ at NLO and $25 \mathrm{MeV}$ up to N3LO. Despite the (apparent) flexibility afforded by the twenty-five LECs (twenty in the charge-independent sector and five more in the charge-dependent one) present in the interaction at N3LO, it has proven rather difficult to extend the fits much beyond $25 \mathrm{MeV}$, while at the same time maintaining $\chi^{2} /$ datum $\lesssim 2$. This may not be surprising, given that in the present EFT formulation the expansion parameter $Q / \Lambda$ with $Q$ and $\Lambda$ being taken, respectively, as the relative momentum and pion mass, is $\approx 0.78$ at a laboratory energy of $25 \mathrm{MeV}$.

A different but potentially related issue is the presence of local minima in the $\chi^{2}$ minimization. There might be more efficient and effective means, like those based on Bayesian methods or machine-learning techniques, to explore the parameter space than standard optimization packages, such as POUNDERS, employed in the present work. An exploratory investigation along these lines is in progress.

In a point of departure from the standard approach, we found it helpful to have LO interactions with projections only in the spin singlet $T=1$ and spin triplet $T=0$ channels, by fixing the associated $C_{01}$ and $C_{10}$ LECs so as to reproduce, respectively, the (large) singlet scattering length and deuteron binding energy. These interactions vanish in odd partial waves (in particular, $P$ waves) that are unconstrained by data (at this order), and, as a consequence, significantly improve the LO description of ground-state energies in $A \geqslant 6$ nuclei. In this respect, we note that a fine tuning of the LECs $C_{01}$ and $C_{10}$ and corresponding cutoffs $R_{1}$ and $R_{0}$ leads to a LO interaction (model o) that correctly reproduces the $n p$ effective range expansions in spin-singlet and spin-triplet channels.

All the N3LO $2 \mathrm{~N}$ interactions overestimate the ${ }^{3} \mathrm{H}$ binding energy except for model $\mathrm{c}$, which leads to an underbinding of about of $40 \mathrm{keV}$ (see Table X). As a result, the LEC $c_{E}$ in the $3 N$ contact interaction accompanying each of these (N3LO) $2 N$ interactions is positive and therefore produces a repulsive contribution for all models except model c; see Table IX part B. However, when $\alpha^{2}$ corrections are ignored in the electromagnetic interaction, the N3LO model c also leads to overbinding in ${ }^{3} \mathrm{H}$ and hence to a repulsive $3 N$ interaction; see again Table IX but now part A. The results in Table X also indicate that the LO and NLO $2 N$ interactions typically overbind (underbind) ${ }^{3} \mathrm{H}$ when the harder (softer) cutoffs with smaller (larger) values for $R_{0}$ and $R_{1}$ are adopted. For the $2 N$ interactions with the softer cutoffs, the need to have an attractive $3 N$ contribution $\left(c_{E}<0\right)$ in order to reproduce the experimental value of the ${ }^{3} \mathrm{H}$ binding energy proves catastrophic in larger nuclei, for example, by wildly overpredicting the ${ }^{16} \mathrm{O}$ binding energy. Indeed, these $2 N$ and $3 N$ models have not been considered in calculations of nuclei with $A \geqslant 16$.

There is a large dependence of the calculated binding energies, particularly in $A \geqslant 16$, on the cutoff of the $3 N$ interaction, the softer cutoff $R_{3}=2.0 \mathrm{fm}$ generally yielding binding energy values closer to experiment; see Table XIII. In one case, model o at LO with $R_{3}=1.0 \mathrm{fm}$ and at NLO with $R_{3}=2.0 \mathrm{fm}$, theory is within less than $2 \%$ of experiment. As matter of fact, the overall picture of nuclear ground-state spectra that emerges from the LO and NLO Hamiltonians corresponding to model o (that is, the model o $2 \mathrm{~N}$ interaction at either LO or NLO in combination with the LO $3 N$ interaction

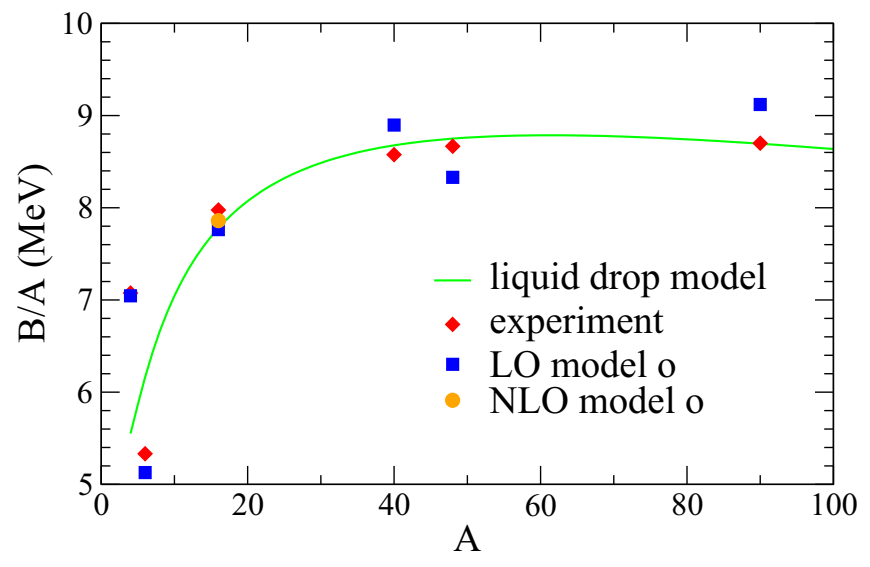

FIG. 6. Binding energy per particle as a function of the atomic number $A$. The liquid drop model results, along with specific experimental values (red symbols) in the cases of $A=4,16,40,48,90$ are shown. Predictions obtained in the present work with the $2 N$ interaction model o at either LO or NLO in combination with the $3 \mathrm{~N}$ interaction at LO, are indicated by the blue (orange) symbols.

having cutoff $R_{3}=1$ and $2 \mathrm{fm}$, respectively) is summarized in Fig. 6, where the predicted binding energies per nucleon are shown as function of the mass number $A$ (only LO results are available in $A \geqslant 40$ ), and are compared to experimental and liquid-drop mass formula values.

The optimization of the two-body $\left(R_{0}\right.$ and $\left.R_{1}\right)$ and threebody $\left(R_{3}\right)$ cutoffs has different motivations. As we have already mentioned, the optimization of $R_{0}$ and $R_{1}$ leads to the correct description of effective range parameters and to an appreciable improvement in the $\chi^{2}$ values obtained in fits to the $2 N$ database. On the other hand, the optimization of $R_{3}$ is aimed at providing a satisfactory description of nuclear binding energies. We have observed that within model o, when $R_{3}$ is set to reproduce reasonably well these energies in the mass range $A \leqslant 16$, the energies for $40 \leqslant A \leqslant 90$ are also reasonably well reproduced. We conclude that this parameter can be used to balance approximately the repulsion-attraction ratio of the nuclear Hamiltonian. A similar situation has been recently observed in the case of bosonic helium clusters [72,73].

Because of the more complicated operator structure of the $2 N$ interactions at N3LO (in particular, the presence of $\mathbf{L}^{2}$ and $(\mathbf{L} \cdot \mathbf{S})^{2}$ terms), these have yet to be implemented in the AFDMC method, and therefore in the present study no N3LO results are reported for $A \geqslant 16$. However, even within the context of calculations based on the NLO $2 \mathrm{~N}$ interactions, there are subleading $3 N$ contact interactions, suppressed by $Q^{2} / \Lambda^{2}$ relative to the LO ones, that need to be accounted for. These terms have a rich operator structure including central, tensor, and spin-orbit-like components but involve a relatively large number (13) of unknown LECs [55]. Arguments based on the large $N_{c}$ expansion allow one to reduce the number of LECs and associated operator structures by ranking their relative importance [56]. Nevertheless, the problem remains of how best to determine these LECs. Two alternative strategies are to constrain them by fitting $3 N$ scattering data (cross sections and polarizations) at low energies or by reproducing 
the energies of low-lying states of selected light nuclei. Both alternatives should be investigated.

\section{ACKNOWLEDGMENTS}

This research is supported by the U.S. Department of Energy, Office of Nuclear Science, under Contracts No. DE-AC05-06OR23177 (A.G. and R.S.) and No. DEAC02-06CH11357 (A.L.), and the U.S. Department of Energy through the FRIB Theory Alliance Award No. DESC0013617 (M.P.). A.L. is also supported by U.S. Department of Energy Early Career Research Program award. The calculations were made possible by grants of computing time from the Argonne Laboratory Computing Resource Center (LCRC), the Argonne Leadership Computing Facility (ALCF) via the 2020/2021 ALCC grant "Chiral Nuclear Interactions from Nuclei to Nucleonic Matter" for the project ChiralNuc, and the National Energy Research Supercomputer Center (NERSC). Computing time on the MARCONI supercomputer at CINECA was also utilized for some of the $A=6$ and $A=16$ calculations.

\section{APPENDIX A: CONFIGURATION-SPACE REPRESENTATION OF THE INTERACTIONS}

The coordinate-space representation of a generic (regularized) term $O(\mathbf{K}, \mathbf{k})$ follows from

$$
O(\mathbf{r})=\int \frac{d \mathbf{k}}{(2 \pi)^{3}} \int \frac{d \mathbf{K}}{(2 \pi)^{3}} e^{i \mathbf{k} \cdot\left(\mathbf{r}^{\prime}+\mathbf{r}\right) / 2} O(\mathbf{K}, \mathbf{k}) e^{i \mathbf{K} \cdot\left(\mathbf{r}^{\prime}-\mathbf{r}\right)}
$$

where $\mathbf{r}$ is the relative position and $\mathbf{K} \longrightarrow \mathbf{p}=-i \nabla^{\prime} \delta\left(\mathbf{r}^{\prime}-\right.$ $\mathbf{r})$, the relative momentum operator. For the momentum-space operator structures present in the contact interactions, we find

$$
\begin{aligned}
1 & \longrightarrow C(r), \\
k^{2} & \longrightarrow-C^{(2)}(r)-\frac{2}{r} C^{(1)}(r), \\
k^{4} & \longrightarrow C^{(4)}(r)+\frac{4}{r} C^{(3)}(r), \\
S_{12}(\mathbf{k}) & \longrightarrow-\left[C^{(2)}(r)-\frac{1}{r} C^{(1)}(r)\right] S_{12}, \\
i \mathbf{S} \cdot(\mathbf{K} \times \mathbf{k}) & \longrightarrow-\frac{1}{r} C^{(1)}(r) \mathbf{L} \cdot \mathbf{S}, \\
(\mathbf{K} \times \mathbf{k})^{2} & \longrightarrow-\frac{1}{r^{2}}\left[C^{(2)}(r)-\frac{1}{r} C^{(1)}(r)\right] \mathbf{L}^{2}+\cdots, \\
{[\mathbf{S} \cdot(\mathbf{K} \times \mathbf{k})]^{2} } & \longrightarrow-\frac{1}{r^{2}}\left[C^{(2)}(r)-\frac{1}{r} C^{(1)}(r)\right](\mathbf{L} \cdot \mathbf{S})^{2}+\cdots,
\end{aligned}
$$

where $C(r)$ is defined in Eq. (2.7) and

$$
C^{(n)}(r)=\frac{d^{n} C(r)}{d r^{n}}
$$

Note that in Eqs. (A7) and (A8) only the terms proportional to $\mathbf{L}^{2}$ and $(\mathbf{L} \cdot \mathbf{S})^{2}$ are retained; the $\cdots$ represent additional terms which either involve terms quadratic in the relative momentum operator or give rise to structures already accounted for. Using the above expressions, the functions $v^{l}(r)$ for the CI terms are obtained as

$$
\begin{aligned}
& v^{c}(r)=v_{\mathrm{LO}}^{c}(r)+C_{1}\left[-C^{(2)}(r)-\frac{2}{r} C^{(1)}(r)\right] \\
& +D_{1}\left[C^{(4)}(r)+\frac{4}{r} C^{(3)}(r)\right] \text {, } \\
& v^{\tau}(r)=v_{\mathrm{LO}}^{\tau}(r)+C_{2}\left[-C^{(2)}(r)-\frac{2}{r} C^{(1)}(r)\right] \\
& +D_{2}\left[C^{(4)}(r)+\frac{4}{r} C^{(3)}(r)\right] \\
& v^{\sigma}(r)=v_{\mathrm{LO}}^{\sigma}(r)+C_{3}\left[-C^{(2)}(r)-\frac{2}{r} C^{(1)}(r)\right] \\
& +D_{3}\left[C^{(4)}(r)+\frac{4}{r} C^{(3)}(r)\right] \text {, } \\
& v^{\sigma \tau}(r)=v_{\mathrm{LO}}^{\sigma \tau}(r)+C_{4}\left[-C^{(2)}(r)-\frac{2}{r} C^{(1)}(r)\right] \\
& +D_{4}\left[C^{(4)}(r)+\frac{4}{r} C^{(3)}(r)\right] \text {, } \\
& v^{t}(r)=-C_{5}\left[C^{(2)}(r)-\frac{1}{r} C^{(1)}(r)\right] \\
& +D_{5}\left[C^{(4)}(r)+\frac{1}{r} C^{(3)}(r)\right. \\
& \left.-\frac{6}{r^{2}} C^{(2)}(r)+\frac{6}{r^{3}} C^{(1)}(r)\right] \text {, } \\
& v^{t \tau}(r)=-C_{6}\left[C^{(2)}(r)-\frac{1}{r} C^{(1)}(r)\right] \\
& +D_{6}\left[C^{(4)}(r)+\frac{1}{r} C^{(3)}(r)\right. \\
& \left.-\frac{6}{r^{2}} C^{(2)}(r)+\frac{6}{r^{3}} C^{(1)}(r)\right] \text {, } \\
& v^{b}(r)=-C_{7} \frac{1}{r} C^{(1)}(r)+D_{7}\left[\frac{1}{r} C^{(3)}(r)\right. \\
& \left.+2 \frac{1}{r^{2}} C^{(2)}(r)-\frac{2}{r^{3}} C^{(1)}(r)\right] \text {, } \\
& v^{b \tau}(r)=D_{8}\left[\frac{1}{r} C^{(3)}(r)+2 \frac{1}{r^{2}} C^{(2)}(r)-\frac{2}{r^{3}} C^{(1)}(r)\right], \\
& v^{b b}(r)=-D_{9} \frac{1}{r^{2}}\left[C^{(2)}(r)-\frac{1}{r} C^{(1)}(r)\right] \text {, } \\
& v^{q}(r)=-D_{10} \frac{1}{r^{2}}\left[C^{(2)}(r)-\frac{1}{r} C^{(1)}(r)\right] \text {, } \\
& v^{q \sigma}(r)=-D_{11} \frac{1}{r^{2}}\left[C^{(2)}(r)-\frac{1}{r} C^{(1)}(r)\right] \text {, }
\end{aligned}
$$

and those for the CD ones are obtained as

$$
v^{T}(r)=C_{0}^{\mathrm{IT}} C(r)+C_{1}^{\mathrm{IT}}\left[-C^{(2)}(r)-\frac{2}{r} C^{(1)}(r)\right],
$$


TABLE XIV. The NLO LECs determined by fitting the $n p$ and $p p$ database up to $15 \mathrm{MeV}$ laboratory energy and the deuteron binding energy.

\begin{tabular}{lccrrr}
\hline \hline Model & $\mathrm{a}$ & $\mathrm{b}$ & $\mathrm{c}$ & $\mathrm{d}$ & \multicolumn{1}{c}{$\mathrm{d}$} \\
\hline$C_{01}\left(\mathrm{fm}^{2}\right)$ & $-0.511051122 \times 10^{1}$ & $-0.515205193 \times 10^{1}$ & $-0.524089036 \times 10^{1}$ & $-0.534645335 \times 10^{1}$ & $-0.514608170 \times 10^{1}$ \\
$C_{10}\left(\mathrm{fm}^{2}\right)$ & $-0.422732988 \times 10^{1}$ & $-0.486213195 \times 10^{1}$ & $-0.147490885 \times 10^{1}$ & $-0.442765927 \times 10^{1}$ & $-0.564430900 \times 10^{1}$ \\
$C_{1}\left(\mathrm{fm}^{4}\right)$ & $-0.112720036 \times 10^{1}$ & $-0.182744818 \times 10^{1}$ & $-0.412069851 \times 10^{1}$ & $-0.483330518 \times 10^{1}$ & $-0.938734989 \times 10^{0}$ \\
$C_{2}\left(\mathrm{fm}^{4}\right)$ & $0.909366063 \times 10^{0}$ & $0.114092429 \times 10^{1}$ & $0.251441807 \times 10^{1}$ & $0.143873251 \times 10^{1}$ & $0.483260368 \times 10^{0}$ \\
$C_{3}\left(\mathrm{fm}^{4}\right)$ & $0.477208278 \times 10^{-1}$ & $0.353463551 \times 10^{0}$ & $0.131550606 \times 10^{1}$ & $0.145157319 \times 10^{1}$ & $0.404430893 \times 10^{0}$ \\
$C_{4}\left(\mathrm{fm}^{4}\right)$ & $-0.475987004 \times 10^{0}$ & $-0.249962307 \times 10^{0}$ & $-0.137446534 \times 10^{0}$ & $0.143861202 \times 10^{1}$ & $-0.531440872 \times 10^{0}$ \\
$C_{5}\left(\mathrm{fm}^{4}\right)$ & $0.494135315 \times 10^{-1}$ & $-0.582318500 \times 10^{-2}$ & $0.688507262 \times 10^{0}$ & $0.347184150 \times 10^{-1}$ & $-0.302484884 \times 10^{0}$ \\
$C_{6}\left(\mathrm{fm}^{4}\right)$ & $-0.846846770 \times 10^{0}$ & $-0.100082249 \times 10^{1}$ & $-0.180046641 \times 10^{1}$ & $-0.125608697 \times 10^{1}$ & $-0.621725001 \times 10^{0}$ \\
$C_{7}\left(\mathrm{fm}^{4}\right)$ & $-0.155550814 \times 10^{1}$ & $-0.138788868 \times 10^{1}$ & $-0.150745124 \times 10^{1}$ & $-0.153475063 \times 10^{1}$ & $-0.136793827 \times 10^{1}$ \\
$C_{0}^{\mathrm{IT}}\left(\mathrm{fm}^{4}\right)$ & $0.190747072 \times 10^{-1}$ & $0.242061782 \times 10^{-1}$ & $0.343911021 \times 10^{-1}$ & $0.488093390 \times 10^{-1}$ & $0.219960910 \times 10^{-1}$ \\
\hline \hline
\end{tabular}

$$
\begin{aligned}
& v_{S}^{\sigma T}(r)=C_{2}^{\mathrm{IT}}\left[-C^{(2)}(r)-\frac{2}{r} C^{(1)}(r)\right], \\
& v^{t T}(r)=-C_{3}^{\mathrm{IT}}\left[C^{(2)}(r)-\frac{1}{r} C^{(1)}(r)\right], \\
& v^{b T}(r)=-C_{4}^{\mathrm{IT}} \frac{1}{r} C^{(1)}(r),
\end{aligned}
$$

where, of course, only the $T=1$ component of the cutoff function enters in the $\mathrm{CD}$ interactions. For ease of presentation, we have singled out the LO terms which only act in $S / T=0 / 1$ and $1 / 0$ channels. They are written as

$$
v_{\mathrm{LO}}^{c}(r)=\frac{3}{16}\left[C_{01} C_{1}(r)+C_{10} C_{0}(r)\right],
$$

$$
\begin{aligned}
& v_{\mathrm{LO}}^{\tau}(r)=\frac{1}{16}\left[C_{01} C_{1}(r)-3 C_{10} C_{0}(r)\right], \\
& v_{\mathrm{LO}}^{\sigma}(r)=\frac{1}{16}\left[-3 C_{01} C_{1}(r)+C_{10} C_{0}(r)\right], \\
& v_{\mathrm{LO}}^{\sigma \tau}(r)=-\frac{1}{16}\left[C_{01} C_{1}(r)+C_{10} C_{0}(r)\right],
\end{aligned}
$$

where there are only two independent LECs, $C_{01}$ and $C_{10}$, and

\begin{tabular}{|c|c|c|c|c|c|}
\hline$C_{01}\left(\mathrm{fm}^{2}\right)$ & $-0.511424764 \times 10^{1}$ & $-0.508230349 \times 10^{1}$ & $-0.503452047 \times 10^{1}$ & $-0.503178655 \times 10^{1}$ & $-0.512268575 \times 10^{1}$ \\
\hline$C_{10}\left(\mathrm{fm}^{2}\right)$ & $-0.425743601 \times 10^{1}$ & $-0.473602278 \times 10^{1}$ & $-0.822959678 \times 10^{0}$ & $-0.376510267 \times 10^{1}$ & $-0.569749961 \times 10^{1}$ \\
\hline$C_{2}\left(\mathrm{fm}^{4}\right)$ & $0.903010818 \times 10^{0}$ & $0.115714920 \times 10^{1}$ & $0.193156537 \times 10^{1}$ & $0.144559465 \times 10^{1}$ & $0.475392426 \times 10^{0}$ \\
\hline$C_{3}\left(\mathrm{fm}^{4}\right)$ & $0.306393229 \times 10^{-1}$ & $0.326127057 \times 10^{0}$ & $0.614523475 \times 10^{0}$ & $0.884483425 \times 10^{0}$ & $0.399019793 \times 10^{0}$ \\
\hline$C_{6}\left(\mathrm{fm}^{4}\right)$ & $-0.849436923 \times 10^{0}$ & $-0.995826514 \times 10^{0}$ & $-0.141944181 \times 10^{1}$ & $-0.505486549 \times 10^{0}$ & $-0.648172781 \times 10^{0}$ \\
\hline$C_{7}\left(\mathrm{fm}^{4}\right)$ & $-0.148260980 \times 10^{1}$ & $-0.121337175 \times 10^{1}$ & $-0.149303317 \times 10^{1}$ & $-0.175175554 \times 10^{1}$ & $-0.134307736 \times 10^{1}$ \\
\hline$D_{1}\left(\mathrm{fm}^{6}\right)$ & $0.362483079 \times 10^{-2}$ & $0.135401332 \times 10^{0}$ & $-0.101959190 \times 10^{1}$ & $-0.119592294 \times 10^{2}$ & $0.376343898 \times 10^{-1}$ \\
\hline$D_{2}\left(\mathrm{fm}^{6}\right)$ & $-0.282368870 \times 10^{-2}$ & $0.587827922 \times 10^{-1}$ & $-0.152848879 \times 10^{0}$ & $-0.314187328 \times 10^{1}$ & $0.185768643 \times 10^{-1}$ \\
\hline$D_{3}\left(\mathrm{fm}^{6}\right)$ & $0.655194092 \times 10^{-2}$ & $0.103792048 \times 10^{0}$ & $0.172566911 \times 10^{0}$ & $-0.159588592 \times 10^{1}$ & $0.208685298 \times 10^{-1}$ \\
\hline$D_{8}\left(\mathrm{fm}^{6}\right)$ & $0.112176977 \times 10^{-1}$ & $-0.427371460 \times 10^{-1}$ & $0.329865430 \times 10^{0}$ & $-0.135461398 \times 10^{1}$ & $-0.146786284 \times 10^{-1}$ \\
\hline$D_{9}\left(\mathrm{fm}^{6}\right)$ & $0.111357163 \times 10^{0}$ & $0.112830624 \times 10^{0}$ & $-0.145021865 \times 10^{0}$ & $0.564521782 \times 10^{1}$ & $0.226506657 \times 10^{-1}$ \\
\hline$D_{10}\left(\mathrm{fm}^{6}\right)$ & $0.325969493 \times 10^{-1}$ & $0.103419159 \times 10^{0}$ & $0.990420811 \times 10^{0}$ & $-0.354055305 \times 10^{0}$ & $0.218482111 \times 10^{-1}$ \\
\hline$D_{11}\left(\mathrm{fm}^{6}\right)$ & $-0.555205059 \times 10^{-1}$ & $-0.962540971 \times 10^{-1}$ & $0.341499700 \times 10^{-2}$ & $0.382300487 \times 10^{1}$ & $0.936405658 \times 10^{-2}$ \\
\hline$C_{0}^{\mathrm{IT}}\left(\mathrm{fm}^{4}\right)$ & $0.616726547 \times 10^{-2}$ & $-0.221853840 \times 10^{-1}$ & $-0.406049402 \times 10^{-1}$ & $-0.127554450 \times 10^{0}$ & $0.713292586 \times 10^{-2}$ \\
\hline$C_{1}^{\mathrm{IT}}\left(\mathrm{fm}^{6}\right)$ & $-0.461868573 \times 10^{-2}$ & $0.852145060 \times 10^{-2}$ & $0.930442901 \times 10^{0}$ & $0.236056581 \times 10^{1}$ & $-0.113805789 \times 10^{-1}$ \\
\hline$C_{2}^{\mathrm{IT}}\left(\mathrm{fm}^{6}\right)$ & $-0.806447857 \times 10^{-2}$ & $-0.276140270 \times 10^{-1}$ & $0.243860719 \times 10^{0}$ & $0.572769698 \times 10^{0}$ & $-0.126174063 \times 10^{-1}$ \\
\hline$C_{3}^{\mathrm{IT}}\left(\mathrm{fm}^{6}\right)$ & $-0.261236310 \times 10^{-1}$ & $-0.156366250 \times 10^{-1}$ & $-0.372938280 \times 10^{-1}$ & $-0.299306179 \times 10^{-1}$ & $0.374105167 \times 10^{-3}$ \\
\hline$C_{4}^{\mathrm{IT}}\left(\mathrm{fm}^{6}\right)$ & $0.156812161 \times 10^{-2}$ & $0.583713002 \times 10^{-1}$ & $0.137474019 \times 10^{0}$ & $-0.218783861 \times 10^{0}$ & $0.298742271 \times 10^{-1}$ \\
\hline
\end{tabular}
the cutoff functions $C_{0}(r)$ and $C_{1}(r)$ are as given in Eq. (2.7).

\section{APPENDIX B: FITTED LECS AT NLO AND N3LO}

Fitted values of the LECs obtained with interactions a-d and $o$ at NLO and N3LO are reported in Tables XIV and XV, respectively.

TABLE XV. The N3LO LECs determined by fitting the $n p$ and $p p$ database up to $25 \mathrm{MeV}$ laboratory energy and the deuteron binding energy. 
[1] S. Weinberg, Phys. Lett. B 251, 288 (1990).

[2] S. Weinberg, Nucl. Phys. B 363, 3 (1991).

[3] C. Ordonez and U. van Kolck, Phys. Lett. B 291, 459 (1992).

[4] C. Ordonez, L. Ray, and U. van Kolck, Phys. Rev. Lett. 72, 1982 (1994).

[5] U. van Kolck, Phys. Rev. C 49, 2932 (1994).

[6] C. Ordonez, L. Ray, and U. van Kolck, Phys. Rev. C 53, 2086 (1996).

[7] D. R. Entem and R. Machleidt, Phys. Rev. C 68, 041001(R) (2003).

[8] E. Epelbaum, W. Glockle, and U. G. Meißner, Nucl. Phys. A 747, 362 (2005).

[9] E. Epelbaum, H. Krebs, and U. G. Meißner, Eur. Phys. J. A 51, 53 (2015).

[10] E. Epelbaum, H. Krebs, and U. G. Meißner, Phys. Rev. Lett. 115, 122301 (2015).

[11] M. Piarulli, L. Girlanda, R. Schiavilla, R. N. Pérez, J. E. Amaro, and E. R. Arriola, Phys. Rev. C 91, 024003 (2015).

[12] D. R. Entem, R. Machleidt, and Y. Nosyk, Phys. Rev. C 96, 024004 (2017).

[13] P. Reinert, H. Krebs, and E. Epelbaum, Eur. Phys. J. A 54, 86 (2018).

[14] V. G. J. Stoks, R. A. M. Klomp, C. P. F. Terheggen, and J. J. de Swart, Phys. Rev. C 49, 2950 (1994).

[15] R. B. Wiringa, V. G. J. Stoks, and R. Schiavilla, Phys. Rev. C 51, 38 (1995).

[16] R. Machleidt, Phys. Rev. C 63, 024001 (2001).

[17] J. W. Chen, G. Rupak, and M. J. Savage, Nucl. Phys. A 653 , 386 (1999).

[18] P. F. Bedaque and U. van Kolck, Annu. Rev. Nucl. Part. Sci. 52, 339 (2002).

[19] H. A. Bethe, Phys. Rev. 76, 38 (1949).

[20] D. B. Kaplan, M. J. Savage, and M. B. Wise, Phys. Lett. B 424, 390 (1998).

[21] D. B. Kaplan, M. J. Savage, and M. B. Wise, Nucl. Phys. B 534, 329 (1998).

[22] H.-W. Hammer, S. König, and U. van Kolck, Rev. Mod. Phys. 92, 025004 (2020).

[23] S. König, H. W. Grießhammer, H. W. Hammer, and U. van Kolck, Phys. Rev. Lett. 118, 202501 (2017).

[24] M. Gattobigio, A. Kievsky, and M. Viviani, Phys. Rev. C 100 034004 (2019).

[25] P. F. Bedaque, H. W. Hammer, and U. van Kolck, Phys. Rev. Lett. 82, 463 (1999).

[26] P. F. Bedaque, H. W. Hammer, and U. van Kolck, Nucl. Phys. A 646, 444 (1999).

[27] E. Braaten and H. W. Hammer, Phys. Rept. 428, 259 (2006).

[28] A. Deltuva, M. Gattobigio, A. Kievsky, and M. Viviani, Phys. Rev. C 102, 064001 (2020).

[29] V. Efimov, Phys. Lett. B 33, 563 (1970).

[30] V. N. Efimov, Sov. J. Nucl. Phys. 12, 589 (1971).

[31] P. Naidon and S. Endo, Rept. Prog. Phys. 80, 056001 (2017).

[32] A. Kievsky, M. Viviani, M. Gattobigio, and L. Girlanda, Phys. Rev. C 95, 024001 (2017).

[33] E. Epelbaum, J. Gegelia, and U. G. Meißner, Nucl. Phys. B 925, 161 (2017).

[34] A. Manohar and H. Georgi, Nucl. Phys. B 234, 189 (1984).

[35] H. Georgi, Phys. Lett. B 298, 187 (1993).

[36] J. Kirscher, H. W. Grießhammer, D. Shukla, and H. M. Hofmann, Eur. Phys. J. A 44, 239 (2010).
[37] V. Lensky, M. C. Birse, and N. R. Walet, Phys. Rev. C 94, 034003 (2016).

[38] E. Epelbaum, J. Gegelia, and U. G. Meißner, Commun. Theor. Phys. 69, 303 (2018).

[39] M. Piarulli, L. Girlanda, R. Schiavilla, A. Kievsky, A. Lovato, L. E. Marcucci, S. C. Pieper, M. Viviani, and R. B. Wiringa, Phys. Rev. C 94, 054007 (2016).

[40] L. Girlanda, A. Kievsky, L. E. Marcucci, and M. Viviani, Phys. Rev. C 102, 064003 (2020).

[41] R. N. Pérez, J. E. Amaro, and E. R. Arriola, Phys. Rev. C 88, 064002 (2013).

[42] R. Navarro Pérez, J. E. Amaro, and E. Ruiz Arriola, Phys. Rev. C 89, 024004 (2014).

[43] R. Navarro Pérez, J. E. Amaro, and E. Ruiz Arriola, Phys. Rev. C 89, 064006 (2014).

[44] M. Kortelainen, T. Lesinski, J. Moré, W. Nazarewicz, J. Sarich, N. Schunck, M. V. Stoitsov, and S. Wild, Phys. Rev. C 82, 024313 (2010).

[45] T. E. O. Ericson and M. Rosa-Clot, Nucl. Phys. A 405, 497 (1983).

[46] N. L. Rodning and L. D. Knutson, Phys. Rev. C 41, 898 (1990).

[47] A. Huber, T. Udem, B. Gross, J. Reichert, M. Kourogi, K. Pachucki, M. Weitz, and T. W. Hansch, Phys. Rev. Lett. 80, 468 (1998).

[48] J. Martorell, D. W. L. Sprung, and D. C. Zheng, Phys. Rev. C 51, 1127 (1995).

[49] J. R. Bergervoet, P. C. van Campen, W. A. van der Sanden, and J. J. de Swart, Phys. Rev. C 38, 15 (1988).

[50] W. A. van der Sanden, A. H. Emmen, and J. J. de Swart, Report No. THEF-NYM-83.11, Nijmegen, 1983 (unpublished); quoted in Ref. [49].

[51] Q. Chen, C. R. Howell, T. S. Carman, W. R. Gibbs, B. F. Gibson, A. Hussein, M. R. Kiser, G. Mertens, C. F. Moore, C. Morris et al., Phys. Rev. C 77, 054002 (2008).

[52] G. A. Miller, M. K. Nefkens, and I. Slaus, Phys. Rep. 194, 1 (1990).

[53] V. G. J. Stoks, R. A. M. Klomp, M. C. M. Rentmeester, and J. J. de Swart, Phys. Rev. C 48, 792 (1993).

[54] F. L. Gross and A. Stadler, Phys. Rev. C 78, 014005 (2008).

[55] L. Girlanda, A. Kievsky, and M. Viviani, Phys. Rev. C 84, 014001 (2011); 102, 019903 (2020).

[56] L. Girlanda, A. Kievsky, M. Viviani, and L. E. Marcucci, Phys. Rev. C 99, 054003 (2019).

[57] A. Kievsky and M. Gattobigio, Few Body Syst. 57, 217 (2016).

[58] A. Kievsky, M. Viviani, D. Logoteta, I. Bombaci, and L. Girlanda, Phys. Rev. Lett. 121, 072701 (2018).

[59] A. Kievsky, S. Rosati, M. Viviani, L. E. Marcucci, and L. Girlanda, J. Phys. G 35, 063101 (2008).

[60] L. E. Marcucci, J. Dohet-Eraly, L. Girlanda, A. Gnech, A. Kievsky, and M. Viviani, Front. Phys. 8, 69 (2020).

[61] A. Gnech, M. Viviani, and L. E. Marcucci, Phys. Rev. C 102, 014001 (2020).

[62] A. Gnech (unpublished).

[63] L. H. Thomas, Phys. Rev. 47, 903 (1935).

[64] K. E. Schmidt and S. Fantoni, Phys. Lett. B 446, 99 (1999).

[65] S. Gandolfi, D. Lonardoni, A. Lovato, and M. Piarulli, Front. Phys. 8, 117 (2020). 
[66] A. Gezerlis, I. Tews, E. Epelbaum, M. Freunek, S. Gandolfi, K. Hebeler, A. Nogga, and A. Schwenk, Phys. Rev. C 90, 054323 (2014).

[67] D. Lonardoni, J. Carlson, S. Gandolfi, J. E. Lynn, K. E. Schmidt, A. Schwenk, and X. B. Wang, Phys. Rev. Lett. 120, 122502 (2018).

[68] D. Lonardoni, S. Gandolfi, J. E. Lynn, C. Petrie, J. Carlson, K. E. Schmidt, and A. Schwenk, Phys. Rev. C 97, 044318 (2018).

[69] S. Gandolfi, A. Lovato, J. Carlson, and K. E. Schmidt, Phys. Rev. C 90, 061306(R) (2014).
[70] L. Contessi, A. Lovato, F. Pederiva, A. Roggero, J. Kirscher, and U. van Kolck, Phys. Lett. B 772, 839 (2017).

[71] A. Ekström, G. R. Jansen, K. A. Wendt, G. Hagen, T. Papenbrock, B. D. Carlsson, C. Forssén, M. Hjorth-Jensen, P. Navrátil, and W. Nazarewicz, Phys. Rev. C 91, 051301(R) (2015).

[72] A. Kievsky, A. Polls, B. Juliá-Díaz, and N. K. Timofeyuk, Phys. Rev. A 96, 040501(R) (2017).

[73] A. Kievsky, A. Polls, B. Juliá-Díaz, N. K. Timofeyuk, and M. Gattobigio, Phys. Rev. A 102, 063320 (2020). 